\title{
An MLP Neural Net with L1 and L2 Regularizers for Real Conditions of Deblurring
}

\author{
Miguel A. Santiago, ${ }^{1}$ Guillermo Cisneros, ${ }^{1}$ and Emiliano Bernués ${ }^{2}$ \\ ${ }^{1}$ Departamento de Señales, Sistemas y Radiocomunicaciones, Escuela Téchica Superior de Ingenieros de Telecomunicación, \\ Universidad Politécnica de Madrid, 28040 Madrid, Spain \\ ${ }^{2}$ Departamento de Ingeniería Electrónica y Comunicaciones, Centro Politécnico Superior, Universidad de Zaragoza, \\ 50018 Zaragoza, Spain \\ Correspondence should be addressed to Miguel A. Santiago, mas@gatv.ssr.upm.es \\ Received 19 March 2010; Revised 2 July 2010; Accepted 6 September 2010 \\ Academic Editor: Enrico Capobianco
}

Copyright ( $) 2010$ Miguel A. Santiago et al. This is an open access article distributed under the Creative Commons Attribution License, which permits unrestricted use, distribution, and reproduction in any medium, provided the original work is properly cited.

Real conditions of deblurring involve a spatially nonlinear process since the borders are truncated, causing significant artifacts in the restored results. Typically, it is assumed to have boundary conditions to reduce ringing; in contrast, this paper proposes a restoration method which simply deals with null borders. We minimize a deterministic regularized function in a Multilayer Perceptron (MLP) with no training and follow a back-propagation algorithm with the L1 and L2 norm-based regularizers. As a result, the truncated borders are regenerated while adapting the center of the image to the optimum linear solution. We report experimental results showing the good performance of our approach in a real model without borders. Even if using boundary conditions, the quality of restoration is comparable to other recent researches.

\section{Introduction}

Image restoration is a classical topic of digital image processing, appearing in many applications such as remote sensing, medical imaging, astronomy, or digital photography [1]. This problem aims to invert a degradation process for recovering the original image, but it is mathematically ill-posed and leads to a highly noise sensitive solution. Consequently, a large number of techniques have been developed to deal with this issue, most of them under the regularization or the Bayesian frameworks (a complete review can found in [2-4]).

The degraded image in those methods comes from the acquisition of a scene in a finite domain (field of view) and exposed to the effects of blurring and additive noise. The image blur is generally modeled as a convolution of the unknown true image with a point spread function (PSF). However, the nonlocal property of the convolution implies that part of the blurred image near the boundary integrates information of the original scenery outside the field of view. This information is not available in the deconvolution process and may cause strong ringing artifacts on the restored image, that is, the well-known boundary problem [5]. Various methods to counteract the boundary effect have been proposed in the literature, making assumptions about the behavior of the original image outside the field of view such as Dirichlet, Neuman, periodic, or other recent conditions in [6-8]. Depending on the boundary assumptions, the blurring matrix adopts a structure with particular computational properties. In fact, the periodic convolution is frequently assumed in the restoration model as the computations can be efficiently performed with block circulant matrices, compared to the block Toeplitz matrixes of the zero-Dirichlet conditions (aperiodic model).

In this paper, we present a restoration method which also starts with a real blurred image in the field of view, but with neither any image information nor prior assumption on the boundary conditions. Furthermore, the objective is not only to improve the restoration on the whole image, but also reconstruct the unknown boundaries of the original image without prior assumption. 


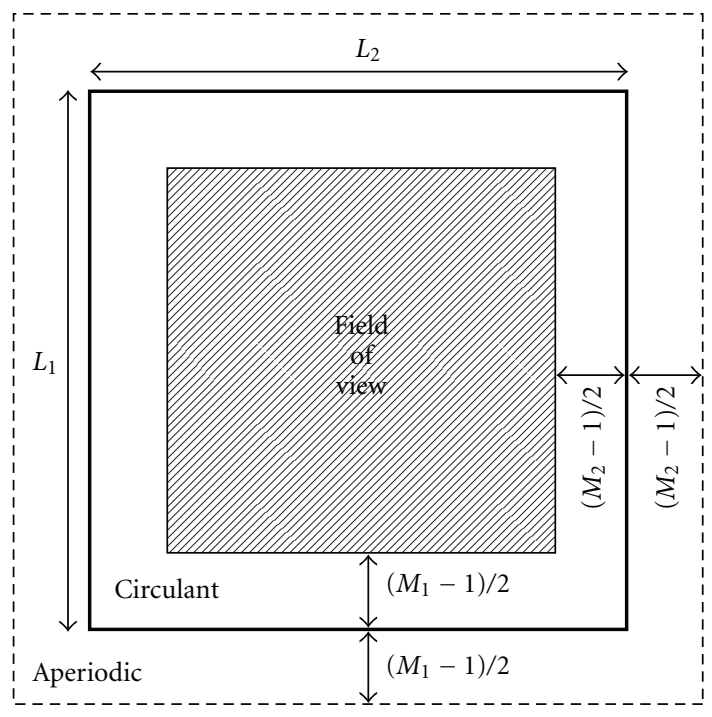

Figure 1: Real observed image which truncates the borders appeared in the circulant and the aperiodic models.

Neural networks are very well suited to combine both processes through the same restoration algorithm, in line with a given adaptation strategy. It could be thought that neural nets are able to learn about the degradation model, and so the borders of the image may be regenerated. For that reason, the algorithm of this paper uses a simple Multilayer Perceptron (MLP) based on the strategy of back-propagation. Others neural-net-based restoration techniques[9-11] have been proposed in the literature with the Hopfield's model however, they tend to be timeconsuming and large scaled. Besides, a Laplace operator is normally used as regularization term in the energy function $\left(\ell_{2}\right.$ regularizer) [9-13], but the success of the TV (total variation) regularization in deconvolution [14-18], also referred as $\ell_{1}$ regularizer in this paper, has motivated its incorporation into our MLP.

A first step of our neural net was given in a previous work [19] using the standard $\ell_{2}$ norm. Here, we propose a newer analysis of the problem on the basis of matrix algebra, using the TV regularizer of [17] and showing a wide range of results. A future research may be addressed to other more effective regularizations terms such as the nonlocal regularization in $[20,21]$.

Let us note that our paper builds somehow on the same algorithmic base presented for the authors in this Journal about the desensitization problem [22]. In fact, our MLP simulates at every iteration an approach to both the degradation (backward) and the restoration (forward) processes, thus extending the same iterative concept but applied to a nonlinear problem. Let us remark that we use here the words "backward" and "forward" in the context of our neural net, which is the opposite sense in a standard image restoration.

This paper is structured as follows. In the next section, we provide a detailed formulation of the problem, establishing naming conventions and the energy functions to be minimized. In Section 3, we present the architecture of the neural net under analysis. Section 4 describes the adjustment of its synaptic weights in every layer for both $\ell_{2}$ and $\ell_{1}$ regularizers and outlines the reconstruction of borders. We present some experimental results in Section 5 and, finally, concluding remarks are given in Section 6.

\section{Problem Formulation}

Let $h(i, j)$ be any generic two-dimensional degradation filter mask (PSF, usually invariant low-pass filter) and $x(i, j)$ the unknown original image, which can be lexicographically represented by the vectors $\mathbf{h}$ and $\mathbf{x}$

$$
\begin{gathered}
\mathbf{h}=\left[h_{1}, h_{2}, \ldots, h_{M}\right]^{T}, \\
\mathbf{x}=\left[x_{1}, x_{2}, \ldots, x_{L}\right]^{T},
\end{gathered}
$$

where $M=\left[M_{1} \times M_{2}\right] \subset \mathfrak{R}^{2}$ and $L=\left[L_{1} \times L_{2}\right] \subset \mathfrak{R}^{2}$ are the respective supports of the PSF and the original image.

A classical formulation of the degradation model (blur and noise) in an image restoration problem is given by

$$
\mathbf{y}=\mathbf{H x}+\mathbf{n},
$$

where $\mathbf{H}$ is the blurring matrix corresponding to the filter mask $\mathbf{h}$ of (1), $\mathbf{y}$ is the observed image (blurred and noisy image), and $\mathbf{n}$ is a sample of a zero mean white Gaussian additive noise of variance $\sigma^{2}$.

The matrix $\mathbf{H}$ can be generally expressed as

$$
\mathbf{H}=\mathbf{T}+\mathbf{B},
$$

where $\mathbf{T}$ has a Toeplitz structure and $\mathbf{B}$, which is defined by the boundary conditions, is often structured, sparse, and low rank. Boundary conditions (BCs) make assumptions about how the observed image behaves outside the field of view (FOV), and they are often chosen for algebraic and computational convenience. The following cases are commonly referenced in literature.

Zero BCs [23], aka Dirichlet, impose a black boundary so that the matrix $\mathbf{B}$ is all zeros, and, therefore, $\mathbf{H}$ has a Toeplitz structure (BTTB). This implies an artificial discontinuity at the borders which can lead to serious ringing effects.

Periodic BCs [23], aka Neumann, assume that the scene can be represented as a mosaic of a single infinite dimensional image, repeated periodically in all directions. The resulting matrix $\mathbf{H}$ is BCCB which can be diagonalized by the unitary discrete Fourier transform and leads to a restoration problem implemented by FFTs. Although computationally convenient, it cannot actually represent a physical observed image and still produces ringing artifacts.

Reflective BCs [24] reflect the image like a mirror with respect to the boundaries. In this case, the matrix $\mathbf{H}$ has a Toeplitz-plus-Hankel structure which can be diagonalized by the orthonormal discrete cosine transformation if the PSF is symmetric. As these conditions maintain the continuity of the graylevel of the image, the ringing effects are reduced in the restoration process. 
Antireflective $B C s$ [7], similarly reflect the image with respect to the boundaries but using a central symmetry instead of the axial symmetry of the reflective BCs. The continuity of the image and the normal derivative are both preserved at the boundary leading to an important reduction of ringing. The structure of $\mathbf{H}$ is Toeplitz-plusHankel and a structured rank 2 matrix, which can be also efficiently implemented if the PSF satisfies a strong symmetry condition.

As a result of these BCs, the matrix product $\mathbf{H x}$ in (2) yields a vector $\mathbf{y}$ of length $\widetilde{L}$, where $\mathbf{H}$ is $\widetilde{L} \times L$ in size and the value of $\widetilde{L}$ depends on the convolution operator. We will mainly analyze the cases of the aperiodic model (linear convolution plus zero BCs) and the circulant model (circular convolution plus periodic BCs) whose parameters are summarized in Table 1. Regarding the reflective and antireflective BCs, they can be managed as an extension of the aperiodic problem, by setting the appropriate boundaries to the original image $\mathbf{x}$.

Then, we come up with a degraded image $\mathbf{y}$ of support $\widetilde{L} \subset \mathfrak{R}^{2}$ with borders derived from the boundary conditions, however, they are not actually present in a real observation. Figure 1 illustrates the borders resulted in the aperiodic and circulant models, and defines the region FOV as

$$
\mathrm{FOV}=\left[\left(L_{1}-M_{1}+1\right) \times\left(L_{2}-M_{2}+1\right)\right] \subset \widetilde{L} .
$$

A real observed image $\mathbf{y}_{\text {real }}$ is, therefore, a truncation of the degradation model up to the size of the FOV support. In our algorithm, we define an image $\mathbf{y}_{\text {tru }}$ which represents this observed image $\mathbf{y}_{\text {real }}$ by means of a truncation on the aperiodic model

$$
\mathbf{y}_{\text {tru }}=\operatorname{trunc}\left\{\mathbf{H}_{\mathbf{a}} \mathbf{x}+\mathbf{n}\right\},
$$

where $\mathbf{H}_{\mathbf{a}}$ is the blurring matrix for the aperiodic model and the operator trunc $\{\cdot\}$ is responsible for removing (zerofixing) the borders appeared due to the boundary conditions, that is to say

$$
\begin{aligned}
y_{\text {tru }}(i, j) & =\operatorname{trunc}\left\{\mathbf{H}_{\mathbf{a}} \mathbf{x}+\left.\mathbf{n}\right|_{(i, j)}\right\} \\
& =\left\{\begin{array}{cc}
\mathbf{y}_{\text {real }}=\mathbf{H}_{\mathbf{a}} \mathbf{x}+\left.\mathbf{n}\right|_{(i, j)} & \forall(i, j) \in \mathrm{FOV} \\
0 & \text { otherwise }
\end{array}\right\} .
\end{aligned}
$$

Dealing with a truncated image like (6) in a restoration problem is an evident source of ringing for the discontinuity at the boundaries. For that reason, this paper aims to provide an image restoration approach to avoid those undesirable ringing artifacts when $\mathbf{y}_{\text {tru }}$ is the observed image. Furthermore, it is also intended to regenerate the truncated borders while adapting the center of the image to the optimum linear solution.

Even if the boundary conditions are maintained in the restoration process, our method is able to reduce the ringing artifacts derived from each boundary discontinuity.

Restoring an image $\mathbf{x}$ is usually an ill-posed or illconditioned problem since either the blurring operator $\mathbf{H}$ does not admit inverse or is nearly singular. Hence, a regularization method should be used in the inversion process for controlling the high sensitivity to the noise. Prominent examples have been presented in the literature by means of the classical Tikhonov regularization

$$
\widehat{\mathbf{x}}=\arg \min _{\mathbf{x}}\left\{\frac{1}{2}\|\mathbf{y}-\mathbf{H} \mathbf{x}\|_{2}^{2}+\frac{\lambda}{2}\|\mathbf{D} \mathbf{x}\|_{2}^{2}\right\},
$$

where $\|\mathbf{z}\|_{2}^{2}=\sum_{i} z_{i}^{2}$ denotes the $\ell_{2}$ norm, $\hat{\mathbf{x}}$ is the restored image and $\mathbf{D}$ is the regularization operator, built on the basis of a high pass filter mask $\mathbf{d}$ of support $N=\left[N_{1} \times\right.$ $\left.\mathrm{N}_{2}\right] \subset \mathfrak{R}^{2}$ and using the same boundary conditions described previously. The first term in (7) is the $\ell_{2}$ residual norm appearing in the least-squares approach and ensures fidelity to data. The second term is the so-called "regularizer" or "side constrain" and captures prior knowledge about the expected behavior of $\mathbf{x}$ through an additional $\ell_{2}$ penalty term involving just the image. The hyperparameter (or regularization parameter) $\lambda$ is a critical value which measures the tradeoff between a good fit and a regularized solution.

Alternatively, the total variation (TV) regularization, proposed by Rudin et al. [25], has become very popular in recent research as result of preserving the edges of objects in the restoration. A discrete version of the TV deblurring problem is given by

$$
\widehat{\mathbf{x}}=\arg \min _{\mathbf{x}}\left\{\frac{1}{2}\|\mathbf{y}-\mathbf{H} \mathbf{x}\|_{2}^{2}+\lambda\|\nabla \mathbf{x}\|_{1}\right\}
$$

where $\|\mathbf{z}\|_{1}$ denotes the $\ell_{1}$ norm (i.e., the sum of the absolute value of the elements) and $\nabla$ stands for the discrete gradient operator. The $\nabla$ operator is defined by the matrices $D^{\xi}$ and $\mathrm{D}^{\mu}$ as

$$
\nabla \mathbf{x}=\left|\mathbf{D}^{\xi} \mathbf{x}\right|+\left|\mathbf{D}^{\mu} \mathbf{x}\right|
$$

built on the basis of the respective masks $\mathbf{d}^{\xi}$ and $\mathbf{d}^{\mu}$ of support $N=\left[N_{1} \times N_{2}\right] \subset \mathfrak{R}^{2}$, which turn out the horizontal and vertical first order differences of the image. Compared to the expression (7), the TV regularization provides a $\ell_{1}$ penalty term which can be thought as a measure of signal variability. Once again, $\lambda$ is the critical regularization parameter to control the weight we assign to the regularizer, relatively to the data misfit term.

In the remainder of the paper, we will refer indistinctly to the $\ell_{2}$ regularizer as the Tikhonov model, and, likewise, the $\ell_{1}$ regularizer may be mentioned as the TV model.

Significant amount of work has been addressed to solve any of the above regularizations and mainly the TV deblurring in recent times. Nonetheless, most of the approaches adopted periodic boundary conditions to cope with the problem on optimal computation basis. We now intend to study $\ell_{1}$ and $\ell_{2}$ regularizers over a suitable restoration approach which manage not only the typical boundary 
TABLE 1: Sizes of the variables involved in the degradation process for the circulant, aperiodic, and real models.

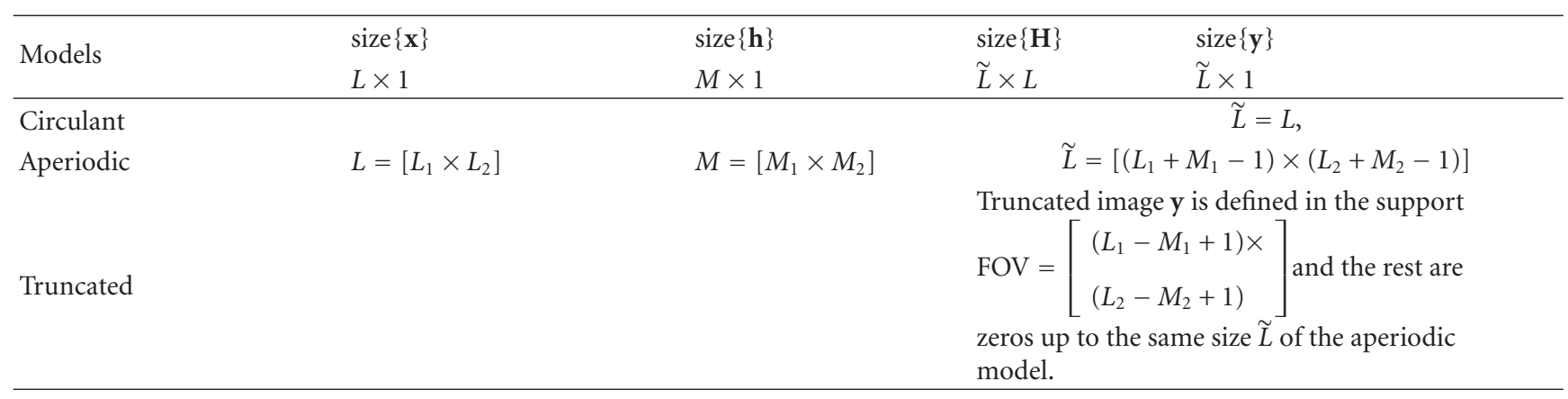

TABLE 2: Size of the variables involved in the restoration process using $\ell_{2}$ and $\ell_{1}$ regularizers, and particularised to the circulant, aperiodic, and real degradation models. The support of the regularisation filters for $\ell_{2}$ and $\ell_{1}$ are equally set to $N=\left[N_{1} \times N_{2}\right]$.

\begin{tabular}{|c|c|c|c|c|}
\hline Regularizer & & $\ell_{2}$ & & $\ell_{1}$ \\
\hline $\operatorname{size}\{\hat{\mathbf{x}}\}$ & $\operatorname{size}\{\mathbf{d}\}$ & $\operatorname{size}\{\mathbf{D x}\}$ & $\operatorname{size}\left\{\mathbf{d}^{\xi}\right\}, \operatorname{size}\left\{\mathbf{d}^{\mu}\right\}$ & $\operatorname{size}\left\{\mathbf{D}^{\xi} \mathbf{x}\right\}, \operatorname{size}\left\{\mathbf{D}^{\mu} \mathbf{x}\right\}$ \\
\hline$L \times 1$ & $N \times 1$ & $U \times L$ & $N \times 1$ & $U \times L$ \\
\hline \multicolumn{5}{|l|}{ Models } \\
\hline Circulant & & $U=L$ & & $U=L$ \\
\hline Aperiodic & & $U=\left[\left(L_{1}+N_{1}-1\right) \times\left(L_{2}+N_{2}-1\right)\right]$ & & $U=\left[\left(L_{1}+N_{1}-1\right) \times\left(L_{2}+N_{2}-1\right)\right]$ \\
\hline Truncated & {$\left[N_{1} \times N_{2}\right]$} & $\begin{array}{l}\text { Truncated image Dx is defined in the } \\
\text { support }\left[\left(L_{1}-N_{1}+1\right) \times\left(L_{2}-N_{2}+1\right)\right] \\
\text { and the rest are zeros up to the same } \\
\text { size } U \text { of the aperiodic model. }\end{array}$ & $N=\left[N_{1} \times N_{2}\right]$ & $\begin{array}{l}\text { Truncated images } \mathbf{D}^{\xi} \mathbf{x} \text { and } \mathbf{D}^{\mu} \mathbf{x} \text { are defined } \\
\text { in the support }\left[\left(L_{1}-N_{1}+1\right) \times\left(L_{2}-N_{2}+1\right)\right] \\
\text { and the rest are zeros up to the same size } U \\
\text { of the aperiodic model. }\end{array}$ \\
\hline
\end{tabular}

conditions, but also the real truncated image as in (5). Consequently, (7) and (8) can redefined as

$$
\begin{aligned}
&\left.\hat{\mathbf{x}}\right|_{\ell 2}=\arg \min _{\mathbf{x}}\{ \frac{1}{2}\left\|\mathbf{y}-\operatorname{trunc}\left\{\mathbf{H}_{\mathbf{a}} \mathbf{x}\right\}\right\|_{2}^{2} \\
&\left.+\frac{\lambda}{2}\left\|\operatorname{trunc}\left\{\mathbf{D}_{\mathbf{a}} \mathbf{x}\right\}\right\|_{2}^{2}\right\}, \\
&\left.\hat{\mathbf{x}}\right|_{\ell 1}=\arg \min _{\mathbf{x}}\left\{\frac{1}{2}\left\|\mathbf{y}-\operatorname{trunc}\left\{\mathbf{H}_{\mathbf{a}} \mathbf{x}\right\}\right\|_{2}^{2}\right. \\
&\left.+\lambda\left\|\operatorname{trunc}\left\{\left|\mathbf{D}_{\mathbf{a}}^{\xi} \mathbf{x}\right|+\left|\mathbf{D}_{\mathbf{a}}^{\mu} \mathbf{x}\right|\right\}\right\|_{1}\right\},
\end{aligned}
$$

where the subscript a denotes the aperiodic formulation of the matrix operator. By removing the operator trunc $\{\cdot\}$ from (10) and (11), and changing it into the specific subscripted operator can be deduced the models for every boundary condition (similar comment can be applied to the remainder of the paper). Table 2 summarizes the dimensions involved in both regularizations taking into account the information provided in Table 1 and the definition of the operator trunc $\{\cdot\}$ in (6).

To go through this problem, we know that neural networks are especially wellsuited as their ability to nonlinear mapping and self-adaptiveness. In fact, the Hopfield network has been used in the literature to solve (7), and recent works are providing neural network solutions to the TV regularization $(8)$ as in $[14,15]$. In this paper, we look for a simple solution to solve both regularizations based on an MLP (Multiplayer Perceptron) with backpropagation.

\section{Definition of the MLP Approach}

Let us build our neural net according to the MLP architecture illustrated in Figure 2. The input layer of the net consists of $\widetilde{L}$ neurons with inputs $y_{1}, y_{2}, \ldots, y_{\tilde{L}}$ being, respectively, the $\tilde{L}$ pixels of the degraded image $\mathbf{y}$. At any generic iteration $m$, the output layer is defined by $L$ neurons whose outputs $\hat{x}_{1}(m), \hat{x}_{2}(m), \ldots, \hat{x}_{L}(m)$ are, respectively, the $L$ pixels of an approach $\hat{\mathbf{x}}(m)$ to the restored image. After $m_{\text {total }}$ iterations, the neural net outcomes the actual restored image $\hat{\mathbf{x}}=$ $\hat{\mathbf{x}}\left(m_{\text {total }}\right)$. On the other hand, the hidden layer consists of two neurons, this being enough to achieve good restoration results while keeping low complexity of the network. In any case, the next analysis will be generalized for any number of hidden layers and any number of neurons per layer.

Whatever the degradation model used in $\mathbf{y}$, the neural net works by simulating at every iteration both an approach to the degradation process (backward) and to the restoration solution (forward), while refining the results progressively at every iteration of the net. However, the input to the net at any iteration is always the degraded image, as no net training is required. Let us recall that we manage "backward" and "forward" concepts in the opposite sense to a standard image restoration because of the architecture of the net.

During the back-propagation process, the network must minimize iteratively a regularized error function which we will precisely set to (10) and (11) in the following sections. Since the trunc $\{\cdot\}$ operator is involved in those expressions, the truncation of the borders is also simulated at every 


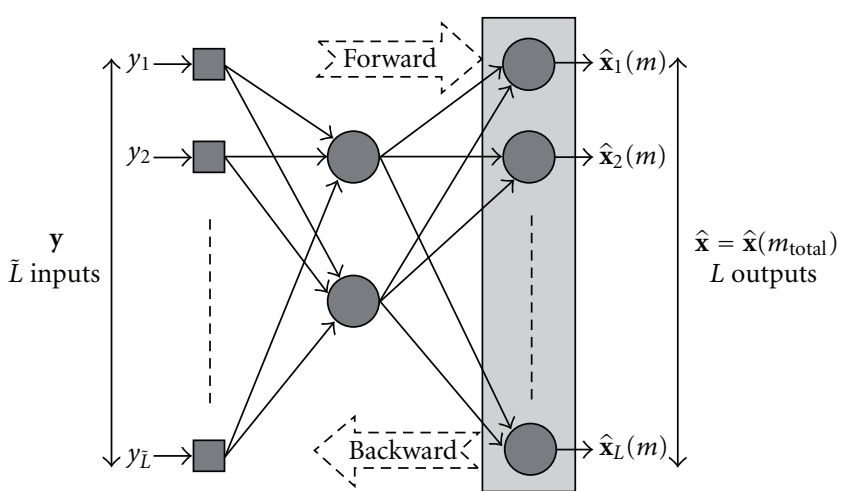

FIGURE 2: MLP scheme adopted for image restoration.

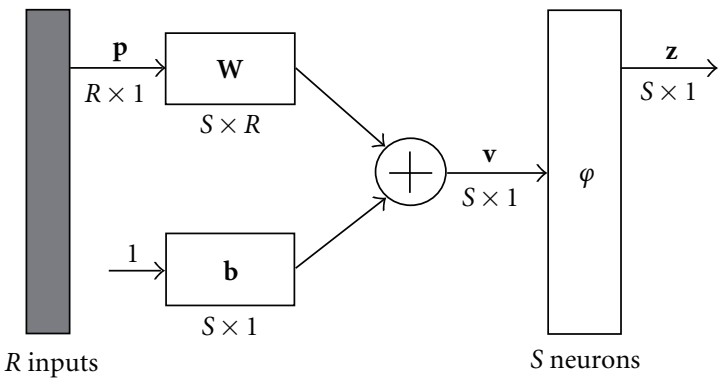

Figure 3: Model of a layer in the MLP

iteration as well as its regeneration, with no a priori knowledge, assumption, or estimation concerning those unknown borders. Consequently, a restored image is obtained in real conditions on the basis of a global energy minimization strategy, with regenerated borders while adapting the centre of the image to the optimum solution and thus making the ringing artifact negligible.

Following a similar naming convention to that adopted in Section 2, let us define any generic layer of the net composed by $R$ inputs and $S$ neurons (outputs) as illustrated in Figure 3.

Where $\mathbf{p}$ is the $R \times 1$ input vector, $\mathbf{W}$ represents the synaptic weight matrix, $S \times R$ in size, and $\mathbf{z}$ is the $S \times 1$ output vector of the layer. The bias vector $\mathbf{b}$ is ignored in our particular implementation. In order to have a differentiable transfer function, a log-sigmoid expression is chosen for $\varphi\{\cdot\}$

$$
\varphi\{\mathbf{v}\}=\frac{1}{1+e^{-v}},
$$

which is defined in the domain $0 \leq \varphi\{\cdot\} \leq 1$.

Then, a layer in the MLP is characterized for

$$
\begin{aligned}
& \mathbf{z}=\varphi\{\mathbf{v}\}, \\
& \mathbf{v}=\mathbf{W} \mathbf{p}+\mathbf{b}=\mathbf{W} \mathbf{p},
\end{aligned}
$$

as $\mathbf{b}=\mathbf{0}$ (vector of zeros). Furthermore, two layers are connected each other verifying that

$$
\mathbf{z}^{\mathbf{i}}=\mathbf{p}^{\mathbf{i}+1}, \quad S^{i}=R^{i+1},
$$

TABLE 3: Summary of dimensions for the output layer.

\begin{tabular}{lcc}
\hline Regularizer & Output layer & $\ell_{1}$ \\
\hline $\operatorname{size}\{\mathbf{p}(m)\}$ & $\mathbf{p}(m)=\mathbf{z}^{i-1}(m) \Rightarrow \operatorname{size}\{\mathbf{p}(m)\}=S^{i-1} \times 1$ \\
$\operatorname{size}\{\mathbf{W}(m)\}$ & $L \times S^{i-1}$ \\
$\operatorname{size}\{\mathbf{v}(m)\}$ & $L \times 1$ \\
$\operatorname{size}\{\mathbf{z}(m)\}$ & $\mathbf{z}(m)=\hat{\mathbf{x}}(m) \Rightarrow \operatorname{size}\{\mathbf{z}(m)\}=L \times 1$ \\
$\operatorname{size}\{\mathbf{e}(m)\}$ & $\widetilde{L} \times 1$ \\
$\operatorname{size}\{\mathbf{r}(m)\}$ & $\operatorname{size}\{\mathbf{D}\}=2 U \times L \Rightarrow$ \\
& $\quad \operatorname{size}\{\mathbf{r}(m)\}=2 U \times 1$ and \\
$\operatorname{size}\{\boldsymbol{\delta}(m)\}$ & $\operatorname{size}\{\mathbf{\Omega}\}=2 U \times 2 U$ \\
& & $L \times 1$
\end{tabular}

where $i$ and $i+1$ are superscripts to denote two consecutive layers of the net. Although this superscripting of layers should be appended to all variables, for notational simplicity we will remove it from all formulae of the paper when deduced by the context.

\section{Adjustment of the Neural Net}

In this section, our purpose is to show the procedure of adjusting the interconnection weights as the MLP iterates. A variant of the well-known algorithm of back-propagation is applied by solving the optimization problems in (10) and (11).

Let $\Delta \mathbf{W}^{i}(m+1)$ be the correction applied to the weight matrix $\mathbf{W}^{i}$ of the layer $i$ at the $(m+1)^{\text {th }}$ iteration. Then,

$$
\Delta \mathbf{W}^{i}(m+1)=-\eta \frac{\partial E(m)}{\partial \mathbf{W}^{i}(m)},
$$

where $E(m)$ stands for the restoration error after $m$ iterations at the output of the net and the constant $\eta$ indicates the learning speed. Let us compute now the so-called gradient matrix $(\partial E(m)) /\left(\partial \mathbf{W}^{i}(m)\right)$ for $\ell_{2}$ and $\ell_{1}$ regularizers in any of the layers of the MLP.

\subsection{Output Layer}

4.1.1. $\ell_{2}$ Regularizer. Defining the vectors $\mathbf{e}(m)$ and $\mathbf{r}(m)$ for the respective error and regularization terms at the output layer after $m$ iterations

$$
\begin{gathered}
\mathbf{e}(m)=\mathbf{y}-\operatorname{trunc}\left\{\mathbf{H}_{\mathbf{a}} \hat{\mathbf{x}}(m)\right\}, \\
\mathbf{r}(m)=\operatorname{trunc}\left\{\mathbf{D}_{\mathbf{a}} \widehat{\mathbf{x}}(m)\right\},
\end{gathered}
$$

we can rewrite the restoration error in a $\ell_{2}$ regularizer problem from (10) as

$$
E(m)=\frac{1}{2}\|\mathbf{e}(m)\|_{2}^{2}+\frac{1}{2} \lambda\|\mathbf{r}(m)\|_{2}^{2} .
$$

Using the matrix chain rule when having a composition on a vector [26], the gradient matrix leads to

$$
\frac{\partial E(m)}{\partial \mathbf{W}(m)}=\frac{\partial E(m)}{\partial \mathbf{v}(m)} \cdot \frac{\partial \mathbf{v}(m)}{\partial \mathbf{W}(m)}=\boldsymbol{\delta}(m) \cdot \frac{\partial \mathbf{v}(m)}{\partial \mathbf{W}(m)} .
$$




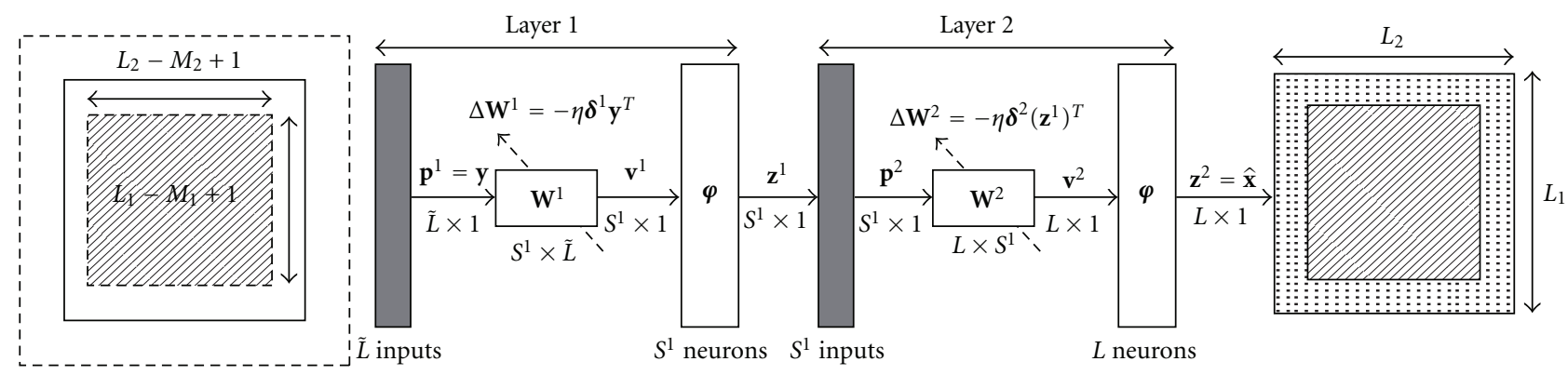

FIgURE 4: MLP algorithm specifically used in the experiments for $J=2$.

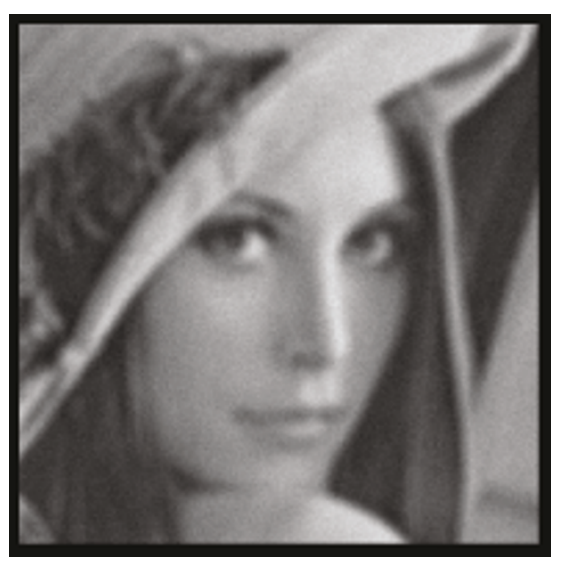

(a)

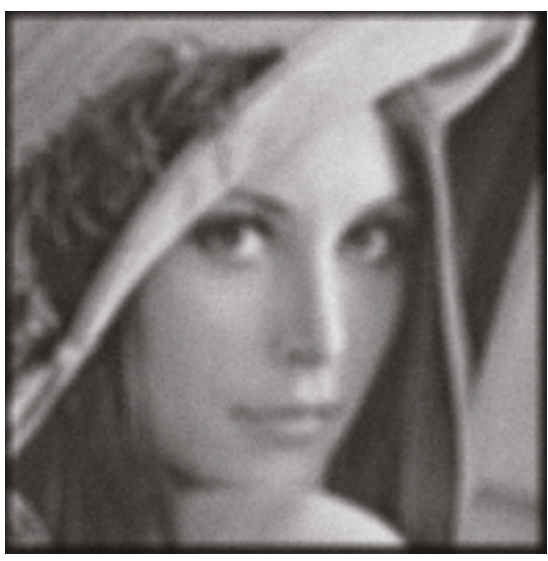

(b)

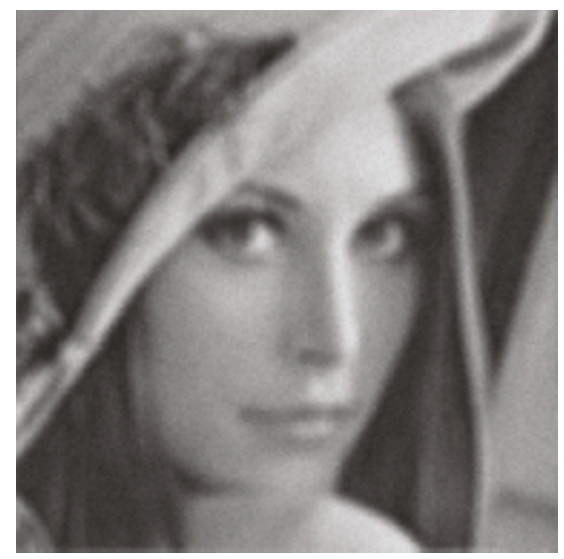

(c)

FIgURE 5: Lena image $256 \times 256$ in size degraded by uniform blur $7 \times 7$ and BSNR $=20 \mathrm{~dB}$ : (a) TRU, (b) APE, and (c) CIR.

where $\boldsymbol{\delta}(m)=(\partial E(m)) /(\partial \mathbf{v}(m))$ is the so-called local gradient vector which again can expanded by the chain rule for vectors [27]

$$
\boldsymbol{\delta}(m)=\frac{\partial \mathbf{z}(m)}{\partial \mathbf{v}(m)} \cdot \frac{\partial E(m)}{\partial \mathbf{z}(m)}
$$

Since $\mathbf{z}$ and $\mathbf{v}$ are elementwise related by the transfer function $\varphi\{\cdot\}$ and thus $\left(\partial z_{i}(m)\right) /\left(\partial v_{j}(m)\right)=0$ for any $i \neq j$, then

$$
\frac{\partial \mathbf{z}(m)}{\partial \mathbf{v}(m)}=\operatorname{diag}\left(\varphi^{\prime}\{\mathbf{v}(m)\}\right)
$$

representing a diagonal matrix whose eigenvalues are computed by the function

$$
\varphi^{\prime}\{\mathbf{v}\}=\frac{e^{-\mathrm{v}}}{\left(1+e^{-\mathrm{v}}\right)^{2}} .
$$

We recall that $\mathbf{z}(m)$ is actually $\widehat{\mathbf{x}}(m)$ in the output layer (see Figure 2). Hence, we can compute the second multiplier of (19) by applying matrix calculus basis over the expressions (16), and (17). A detailed computation can be found in the appendix and leads to

$$
\frac{\partial E(m)}{\partial \mathbf{z}(m)}=\frac{\partial E(m)}{\partial \hat{\mathbf{x}}(m)}=-\mathbf{H}_{\mathbf{a}}^{T} \mathbf{e}(m)+\lambda \mathbf{D}_{\mathbf{a}}^{T} \mathbf{r}(m) .
$$

According to the Tables 1 and 2, $(\partial E(m)) /(\partial \mathbf{z}(m))$ represents a vector of size $L \times 1$. When combining with the diagonal matrix of (20), we can write

$$
\boldsymbol{\delta}(m)=\varphi^{\prime}\{\mathbf{v}(m)\} \circ\left(-\mathbf{H}_{\mathbf{a}}^{T} \mathbf{e}(m)+\lambda \mathbf{D}_{\mathbf{a}}^{T} \mathbf{r}(m)\right)
$$

where $\circ$ denotes the Hadamard (elementwise) product.

To complete the analysis of the gradient matrix, we have to compute the term $(\partial \mathbf{v}(m)) /(\partial \mathbf{W}(m))$. Based on the layer definition in the MLP (13), we obtain

$$
\frac{\partial \mathbf{v}(m)}{\partial \mathbf{W}(m)}=\frac{\partial \mathbf{W}(m) \mathbf{p}(m)}{\partial \mathbf{W}(m)}=\mathbf{p}^{T}(m)
$$

which in turns corresponds to the output of the previous connected hidden layer, that is to say

$$
\frac{\partial \mathbf{v}(m)}{\partial \mathbf{W}(m)}=\left(\mathbf{z}^{i-1}(m)\right)^{T}
$$




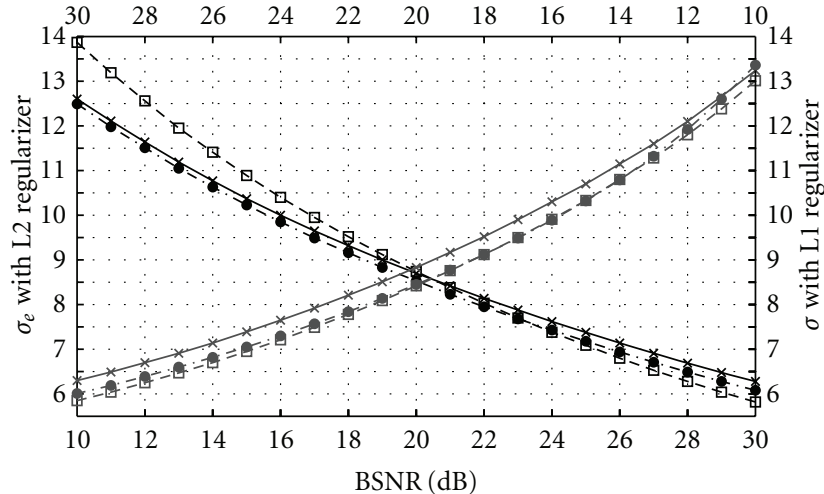

$\begin{array}{ll}* & \text { TRU } \\ -\bullet- & \text { APE } \\ -\bullet & \text { CIR }\end{array}$

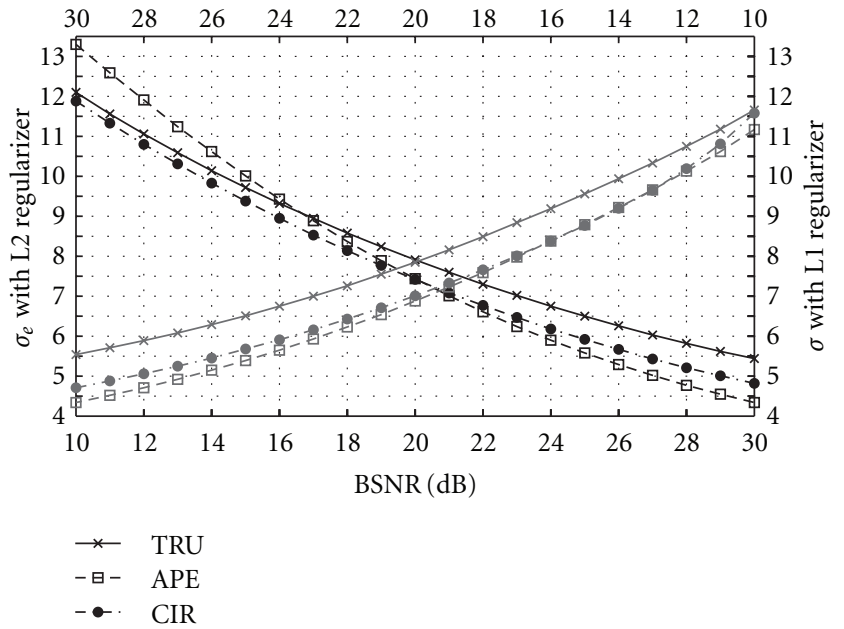

(b)

FIgURE 6: Restoration error $\sigma_{e}$ for $\ell_{2}$ and $\ell_{1}$ regularizers using TRU, APE, and CIR degradation models: (a) filter $\mathbf{h}_{\mathbf{1}}$ (b) filter $\mathbf{h}_{\mathbf{2}}$.

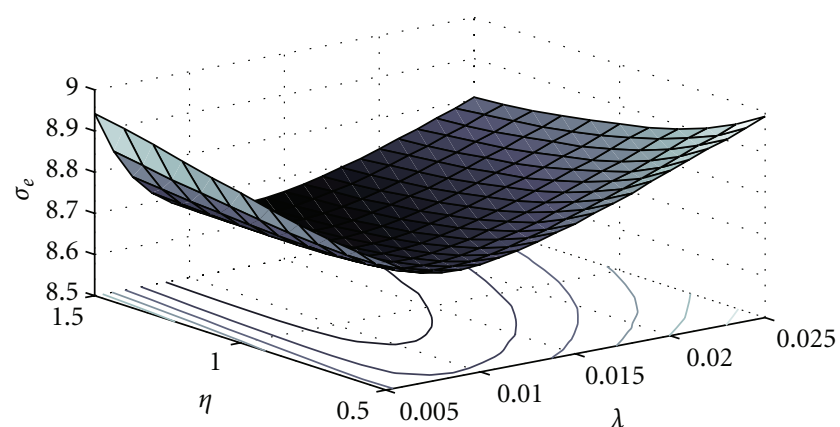

Figure 7: Sensitivity of $\sigma_{\mathrm{e}}$ to $\eta$ and $\lambda$.

Putting together all the results into the incremental weight matrix $\Delta \mathbf{W}(m+1)$, we have

$$
\begin{aligned}
\Delta \mathbf{W}(m+1)= & -\eta \boldsymbol{\delta}(m)\left(\mathbf{z}^{i-1}(m)\right)^{T} \\
= & -\eta\left[\varphi^{\prime}\{\mathbf{v}(m)\} \circ\left(-\mathbf{H}_{\mathbf{a}}^{T} \mathbf{e}(m)+\lambda \mathbf{D}_{\mathbf{a}}^{T} \mathbf{r}(m)\right)\right] \\
& \times\left(\mathbf{z}^{i-1}(m)\right)^{T} .
\end{aligned}
$$

4.1.2. $\ell_{1}$ Regularizer. In the light of the above regularizer, let us also define analogous error and regularization terms with respect to $(8)$

$$
\begin{gathered}
\mathbf{e}(m)=\mathbf{y}-\operatorname{trunc}\left\{\mathbf{H}_{\mathbf{a}} \widehat{\mathbf{x}}(m)\right\}, \\
\mathbf{r}(m)=\operatorname{trunc}\left\{\left|\mathbf{D}_{\mathbf{a}}^{\xi} \widehat{\mathbf{x}}(m)\right|+\left|\mathbf{D}_{\mathbf{a}}^{\mu} \widehat{\mathbf{x}}(m)\right|\right\} .
\end{gathered}
$$

With these definitions, $E(m)$ can be written in a compact notation as

$$
E(m)=\frac{1}{2}\|\mathbf{e}(m)\|_{2}^{2}+\lambda\|\mathbf{r}(m)\|_{1} .
$$

If we aimed to compute the gradient matrix $\partial E(m) /$ $\partial \mathbf{W}_{\mathbf{i}}(m)$ with (29), we would find out a challenging nonlinear optimization problem that is caused by the nondifferentiability of the $\ell_{1}$ norm. One approach to overcome this challenge comes from

$$
\begin{aligned}
\|\mathbf{r}(m)\|_{1} & \approx \operatorname{TV}\{\hat{\mathbf{x}}(m)\} \\
& =\sum_{k} \sqrt{\left(\mathbf{D}_{\mathbf{a}}^{\xi} \hat{\mathbf{x}}(m)\right)_{k}^{2}+\left(\mathbf{D}_{\mathbf{a}}^{\mu} \hat{\mathbf{x}}(m)\right)_{k}^{2}+\varepsilon},
\end{aligned}
$$

where TV stands for the well-known total variation regularizer and $\varepsilon>0$ is a constant to avoid singularities when minimizing. Both products $\mathbf{D}_{\mathbf{a}}^{\xi} \widehat{\mathbf{x}}(m)$, and $\mathbf{D}_{\mathbf{a}}^{\mu} \widehat{\mathbf{x}}(m)$ are subscripted by $k$ meaning the $k$ th element of the respective $U \times 1$ sized vector (see Table 2 ). It should be mentioned that $\ell_{1}$ norm and TV regularizations are quite often used as the same in the literature. But the distinction between these two regularizers should be kept in mind since, at least in deconvolution problems, TV leads to significant better results as illustrated in [16].

Bioucas-Dias et al. $[16,17]$ proposed an interesting formulation of the total variation problem by applying majorization-minimization algorithms (MM). It leads to a quadratic bound function for TV regularizer, which thus results in solving a linear system of equations. Likewise, we adopt that quadratic majorizer in our particular implementation as

$$
\operatorname{TV}\{\widehat{\mathbf{x}}(m)\} \leq Q_{\mathrm{TV}}\{\widehat{\mathbf{x}}(m)\}=\hat{\mathbf{x}}^{T}(m) \mathbf{D}_{\mathbf{a}}^{T} \boldsymbol{\Omega}(m) \mathbf{r}(m)+K,
$$


where $K$ is an irrelevant constant, the involved matrixes are defined as

$$
\begin{gathered}
\mathbf{D}_{\mathbf{a}}=\left[\left(\mathbf{D}_{\mathbf{a}}^{\xi}\right)^{T}\left(\mathbf{D}_{\mathbf{a}}^{\mu}\right)^{T}\right]^{T}, \\
\boldsymbol{\Omega}(m)=\left[\begin{array}{cc}
\boldsymbol{\Lambda}(m) & 0 \\
0 & \boldsymbol{\Lambda}(m)
\end{array}\right],
\end{gathered}
$$

with

$$
\boldsymbol{\Lambda}(m)=\operatorname{diag}\left(\frac{1}{2 \sqrt{\left(\mathbf{D}_{\mathbf{a}}^{\xi} \widehat{\mathbf{x}}(m)\right)^{2}+\left(\mathbf{D}_{\mathbf{a}}^{\mu} \widehat{\mathbf{x}}(m)\right)^{2}+\varepsilon}}\right),
$$

and the regularization term $\mathbf{r}(m)$ of $(28)$ is reformulated

$$
\mathbf{r}(m)=\operatorname{trunc}\left\{\mathbf{D}_{\mathbf{a}} \hat{\mathbf{x}}(m)\right\},
$$

such that the operator trunc $\{\cdot\}$ works by applying it individually for $\mathbf{D}_{\mathbf{a}}^{\xi}$ and $\mathbf{D}_{\mathbf{a}}^{\mu}$ (see Table 2) and merging later as indicated in the definition of (32).

Finally, we can rewrite the restoration error $E(m)$ as

$$
E(m)=\frac{1}{2}\|\mathbf{e}(m)\|_{2}^{2}+\lambda Q_{\mathrm{TV}}\{\widehat{\mathbf{x}}(m)\} .
$$

The same steps as in $\ell_{2}$ regularizer can be followed now to compute the gradient matrix. When we come to resolve the differentiation $(\partial E(m)) /(\partial \mathbf{z}(m))$, we take advantage of the quadratic properties of the expression (31) and the derivation of (22) so as to obtain

$$
\frac{\partial E(m)}{\partial \mathbf{z}(m)}=\frac{\partial E(m)}{\partial \widehat{\mathbf{x}}(m)}=-\mathbf{H}_{\mathbf{a}}^{T} \mathbf{e}(m)+\lambda \mathbf{D}_{\mathbf{a}}^{T} \boldsymbol{\Omega}(m) \mathbf{r}(m) .
$$

It can be deduced as an extension of the $\ell_{2}$ solution when using the first-order differences operator $\mathbf{D}_{\mathbf{a}}$ of (32) and incorporating the weigh matrix $\mathbf{\Omega}(m)$. In fact, this spatially varying matrix is responsible for the smoothness or sharpness (presence of edges) of the solution depending on the local differences of the image.

The remaining steps for the analysis of $(\partial E(m)) /(\partial \mathbf{W}(m))$ are identical to the previous section and yield a local gradient vector as

$$
\boldsymbol{\delta}(m)=\varphi^{\prime}\{\mathbf{v}(m)\} \circ\left(-\mathbf{H}_{\mathbf{a}}^{T} \mathbf{e}(m)+\lambda \mathbf{D}_{\mathbf{a}}^{T} \boldsymbol{\Omega}(m) \mathbf{r}(m)\right),
$$

Finally, we come to the following variation of the weight matrix

$$
\begin{aligned}
\Delta \mathbf{W}(m+1) \\
=-\eta \boldsymbol{\delta}(m)\left(\mathbf{z}^{i-1}(m)\right)^{T} \\
=-\eta\left[\varphi^{\prime}\{\mathbf{v}(m)\} \circ\left(-\mathbf{H}_{\mathbf{a}}^{T} \mathbf{e}(m)+\lambda \mathbf{D}_{\mathbf{a}}^{T} \mathbf{\Omega}(m) \mathbf{r}(m)\right)\right] \\
\quad \times\left(\mathbf{z}^{i-1}(m)\right)^{T} .
\end{aligned}
$$

4.2. Any $i$ Hidden Layer. If we set superscripting for the gradient matrix (18) over any $i$ hidden layer of the MLP, we obtain

$$
\frac{\partial E(m)}{\partial \mathbf{W}^{i}(m)}=\frac{\partial E(m)}{\partial \mathbf{v}^{i}(m)} \cdot \frac{\partial \mathbf{v}^{i}(m)}{\partial \mathbf{W}^{\mathbf{i}}(m)}=\boldsymbol{\delta}^{i}(m) \cdot \frac{\partial \mathbf{v}^{i}(m)}{\partial \mathbf{W}^{i}(m)},
$$

and taking what was already demonstrated in (25), then

$$
\frac{\partial E(m)}{\partial \mathbf{W}^{i}(m)}=\boldsymbol{\delta}^{i}(m)\left(\mathbf{z}^{i-1}(m)\right)^{T}
$$

Let us expand the local gradient $\boldsymbol{\delta}^{i}(m)$ by means of the chain rule for vectors as follows:

$$
\boldsymbol{\delta}^{i}(m)=\frac{\partial E(m)}{\partial \mathbf{v}^{i}(m)}=\frac{\partial \mathbf{z}^{i}(m)}{\partial \mathbf{v}^{i}(m)} \cdot \frac{\partial \mathbf{v}^{i+1}(m)}{\partial \mathbf{z}^{i}(m)} \cdot \frac{\partial E(m)}{\partial \mathbf{v}^{i+1}(m)},
$$

where $\left(\partial \mathbf{z}^{i}(m)\right) /\left(\partial \mathbf{v}^{i}(m)\right)$ is the same diagonal matrix (20), whose eigenvalues are represented by $\varphi^{\prime}\left\{\mathbf{v}^{i}(m)\right\}$, and $(\partial E(m)) /\left(\partial \mathbf{v}^{i+1}(m)\right)$ denotes the local gradient $\boldsymbol{\delta}^{i+1}(m)$ of the following connected layer. With respect to the term $\left(\partial \mathbf{v}^{i+1}(m)\right) /\left(\partial \mathbf{z}^{i}(m)\right)$, it can be immediately derived from the MLP definition of (13) that

$$
\begin{aligned}
\frac{\partial \mathbf{v}^{i+1}(m)}{\partial \mathbf{z}^{i}(m)} & =\frac{\partial \mathbf{W}^{i+1}(m) \mathbf{p}^{i+1}(m)}{\partial \mathbf{z}^{i}(m)} \\
& =\frac{\partial \mathbf{W}^{i+1}(m) \mathbf{z}^{i}(m)}{\partial \mathbf{z}^{i}(m)}=\left(\mathbf{W}^{i+1}(m)\right)^{T} .
\end{aligned}
$$

Consequently, we come to

$$
\boldsymbol{\delta}^{i}(m)=\operatorname{diag}\left(\varphi^{\prime}\left\{\mathbf{v}^{i}(m)\right\}\right)\left(\mathbf{W}^{i+1}(m)\right)^{T} \boldsymbol{\delta}^{i+1}(m),
$$

which can be simplified after verifying that $\left(\mathbf{W}^{i+1}(m)\right)^{T} \boldsymbol{\delta}^{i+1}(m)$ stands for a $R^{i+1} \times 1=S^{i} \times 1$ vector

$$
\boldsymbol{\delta}^{i}(m)=\varphi^{\prime}\left\{\mathbf{v}^{i}(m)\right\} \circ\left(\left(\mathbf{W}^{i+1}(m)\right)^{T} \boldsymbol{\delta}^{i+1}(m)\right)
$$

We finally provide an equation to compute the incremental weight matrix $\Delta \mathbf{W}^{i}(m+1)$ for any $i$ hidden layer

$$
\begin{aligned}
\Delta \mathbf{W}^{i}(m+1)= & -\eta \boldsymbol{\delta}^{i}(m)\left(\mathbf{z}^{i-1}(m)\right)^{T} \\
=- & \eta\left[\varphi^{\prime}\left\{\mathbf{v}^{i}(m)\right\} \circ\left(\left(\mathbf{W}^{i+1}(m)\right)^{T} \boldsymbol{\delta}^{i+1}(m)\right)\right], \\
& \times\left(\mathbf{z}^{i-1}(m)\right)^{T}
\end{aligned}
$$

which is mainly based on the local gradient $\boldsymbol{\delta}^{i+1}(m)$ of the following connected layer $i+1$.

It is worthy to mention that we have not made any distinction between regularizers. Precisely, the term $\boldsymbol{\delta}^{i+1}(m)$ is in charge of propagating which regularizer is used when processing the output layer. 


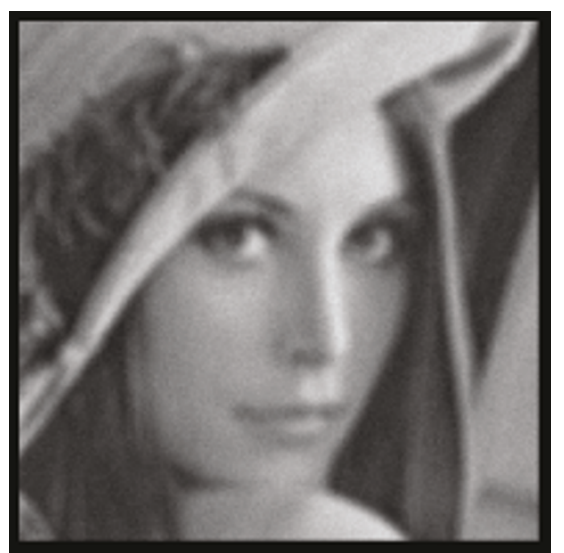

(a)

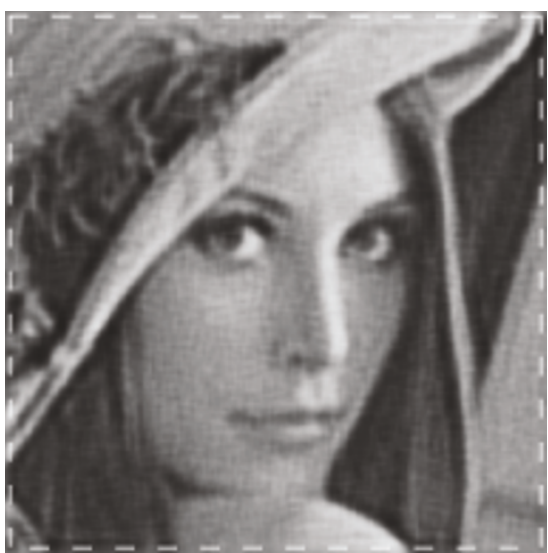

(b)

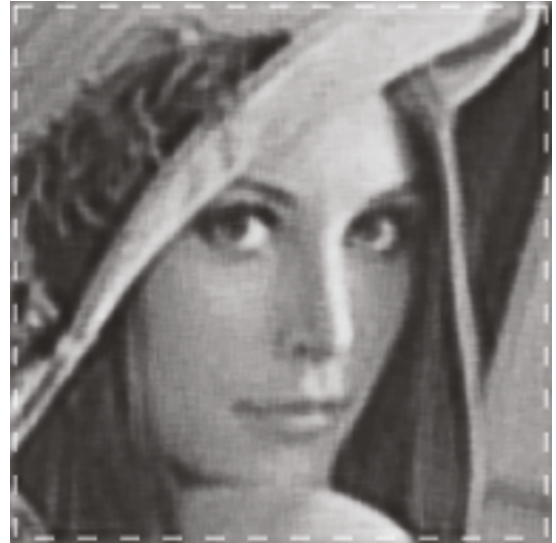

(c)

FIgURE 8: Restoration results from the Lena degraded image by uniform blur $7 \times 7$, BSNR $=20 \mathrm{~dB}$ and TRU model (a). Respectively for $\ell_{2}$ and $\ell_{1}$, the restored images are shown in (b) and (c). A broken white line highlights the regeneration of borders.

Initialization: $\mathbf{p}^{1}=\mathbf{y}$ forall $m$ and $\mathbf{W}^{i}(0)=\mathbf{0} 1 \leq i \leq J$
(1) $m:=0$
(2) while StopRule not satisfied do
(3) for $i:=1$ to $J$ do $/ *$ Forward */
(4) $\quad \mathbf{v}^{i}:=\mathbf{W}^{i} \mathbf{p}^{i}$
(5) $\quad \mathbf{z}^{i}:=\varphi\left\{\mathbf{v}^{i}\right\}$
(6) end for $/ * \widehat{\mathbf{x}}(m):=\mathbf{z}^{J} * /$
(7) for $i:=J$ to 1 do $/ *$ Backward */
(8) if $i=J$ then $/ *$ Output layer */
(9) if $\ell=\ell_{2}$ then
(10) Compute $\boldsymbol{\delta}^{J}(m)$ from (23)
(11) Compute $E(m)$ from (17)
(12) elseif $\ell=\ell_{1}$ then
(13) Compute $\boldsymbol{\delta}^{J}(m)$ from (37)
(14) Compute $E(m)$ from (35)
(15) end if
(16) else
(17) $\boldsymbol{\delta}^{i}(m):=\varphi^{\prime}\left\{\mathbf{v}^{i}(m)\right\} \circ\left(\left(\mathbf{W}^{i+1}(m)\right)^{T} \boldsymbol{\delta}^{i+1}(m)\right)$
(18) end if
(19) $\Delta \mathbf{W}^{i}(m+1):=-\eta \boldsymbol{\delta}^{i}(m)\left(\mathbf{z}^{i-1}(m)\right)^{T}$
(20) $\quad \mathbf{W}^{i}(m+1):=\mathbf{W}^{i}(m)+\Delta \mathbf{W}^{i}(m+1)$
(21) end for
(22) $m:=m+1$
(23) end while $/ * \widehat{\mathbf{x}}:=\widehat{\mathbf{x}}\left(m_{\text {total }}\right) * /$

Algorithm 1: MLP with $\ell$ regularizer.

4.3. Algorithm. As described in Section 3, our MLP neural net works by performing a couple of forward and backward processes at every iteration $m$. Firstly, the whole set of connected layers propagate the degraded image $\mathbf{y}$ from the input to the output layers by means of (13). Afterwards, the new synaptic weigh matrixes $\mathbf{W}^{i}(m+1)$ are recalculated from right to left according to the expressions of $\Delta \mathbf{W}^{i}(m+1)$ for every layer.

The previous pseudocode summarizes our proposed algorithm for $\ell_{1}$ and $\ell_{2}$ regularizers in a MLP of $J$ layers.
There, StopRule denotes a condition such that either the number of iterations is more than a maximum or the error $E(m)$ converges, and thus, the error change $\Delta E(m)$ is less than a threshold, or, even, this error $E(m)$ starts to increase. If one of these conditions comes true, the algorithm concludes and the final outgoing image is just the restored image $\widehat{\mathbf{x}}:=$ $\hat{\mathbf{x}}\left(m_{\text {total }}\right)$.

4.4. Regeneration of Borders. If we particularize the algorithm for two layers $J=2$, we come to a MLP scheme such as illustrated in Figure 4. It is worthy to emphasize how the borders are regenerated at any iteration of the net, from a real image of support FOV(4) to the restored image of size $L=\left[L_{1} \times L_{2}\right]$ (recall that the remainder of pixels in $\mathbf{y}$ was zerofixed). Additionally, we will observe in Section 5 how the boundary artifacts are removed from the restored image based on the energy minimization $E(m)$, but they are critical, however, for other methods of the literature.

4.5. Adjustment of $\lambda$ and $\eta$. In the image restoration field, it is wellknown how important the parameter $\lambda$ becomes. In fact, too small values of $\lambda$ yield overly oscillatory estimates owing to either noise or discontinuities, too large values of $\lambda$ yield over smoothed estimates.

For that reason, the literature has given significant attention to it with popular approaches such as the unbiased predictive risk estimator (UPRE), the generalized cross validation (GCV), or the L-curve method; see [28] for an overview and references. Most of them were particularized for a Tikhonov regularizer, but lately researches aim to provide solutions for TV regularization. Specifically, the Bayesian framework leads to successful approaches in this field.

Since we do not have yet a particular algorithm to adjust $\lambda$ in the MLP, then we will take solutions coming from the Bayesian state-of-art. However, let us recall that most of them are developed when assuming a circulant model for the observed image and, thus, not optimized for the aperiodic 


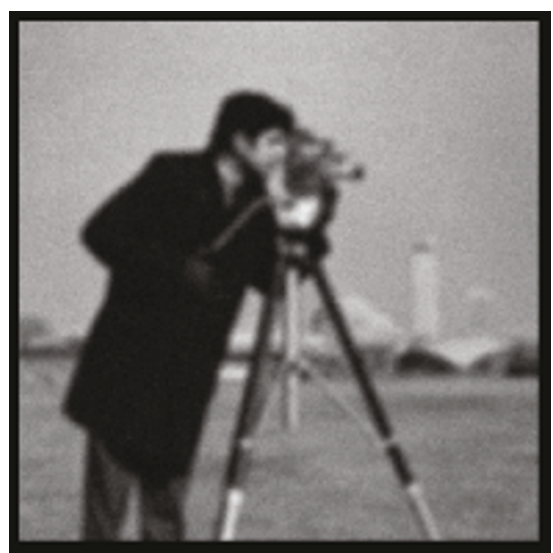

(a)

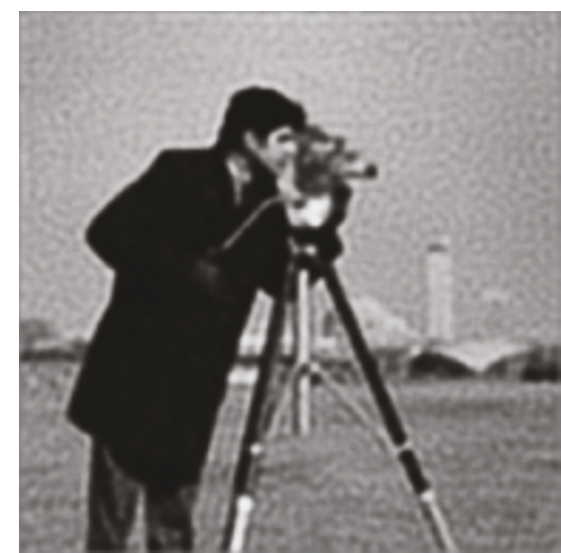

(b)

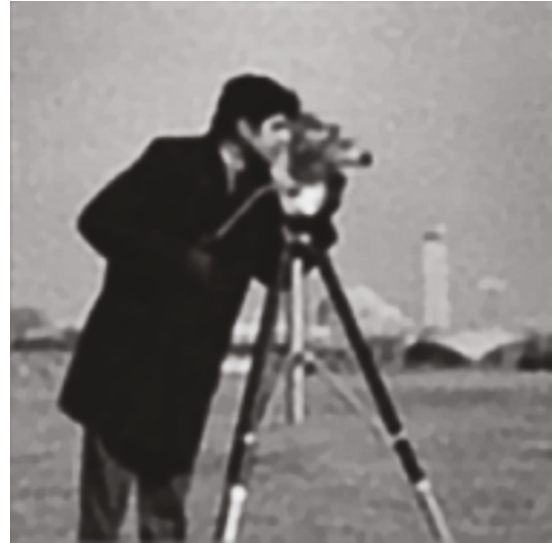

(c)

FIGURE 9: Restoration results from the Cameraman degraded image by Gaussian blur $7 \times 7$, BSNR $=20$ dB and TRU model (a). Respectively for $\ell_{2}$ and $\ell_{1}$, the restored images are shown in (b) $\sigma_{e}=16.08$ and (c) $\sigma_{e}=15.74$.

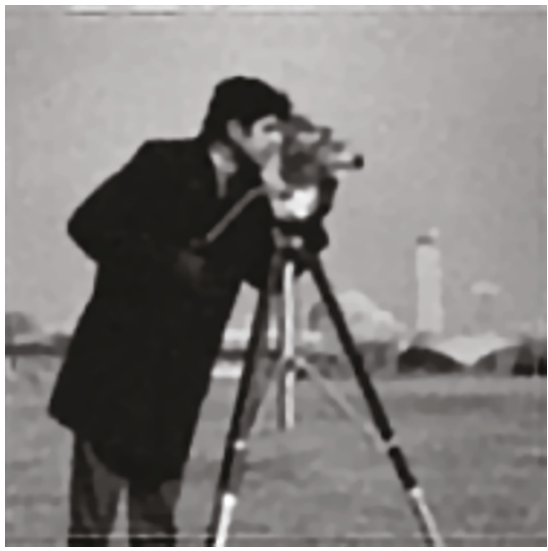

FIgURE 10: Artifacts appeared when removing the boundary conditions, cropping the center, in a MM1 algorithm. With zeros outside, the restoration is completely corrupted.

or truncated models of this paper. We will summarize the equations which have better adapted to our neural net in the following subsections.

It is important to note that $\lambda$ must be computed for every iteration $m$ of the MLP. Consequently, as the solution $\widehat{\mathbf{x}}(m)$ approaches to the final restored image, the regularization parameter $\lambda(m)$ also tends to its optimum value. So, in order to obtain better results, a second computation of the whole neural net will be executed fixing the previous $\lambda\left(m_{\text {total }}\right)$.

Regarding the learning speed $\eta$, we will empirically observe in Section 5 that shows lower sensitivity compared to $\lambda$. In fact, its main purpose is to speed up or slow down the convergence of the algorithm. Then, for the sake of simplicity, we assume $\eta=1$ or $\eta=2$ depending on the size of the image.

4.5.1. $\ell_{2}$ Regularizer. Molina et al. [29] deal with the estimation of the hyperparameters $\alpha$ and $\beta(\lambda=\alpha / \beta)$ under a Bayesian paradigm for a $\ell_{2}$ regularization as in
(7). So, assuming a simultaneous autoregressive (SAR) prior distribution for the original image, we can express their results in terms of our variables as

$$
\begin{aligned}
& \frac{1}{\alpha(m)}=\frac{1}{\widetilde{L}}\|\mathbf{r}(m)\|_{2}^{2}+\frac{1}{L} \operatorname{trace}\left\{\mathbf{Q}^{-1}(\alpha, \beta) \mathbf{D}_{\mathbf{a}}^{\mathrm{T}} \mathbf{D}_{\mathbf{a}}\right\}, \\
& \frac{1}{\beta(m)}=\frac{1}{\widetilde{L}}\|\mathbf{e}(m)\|_{2}^{2}+\frac{1}{L} \operatorname{trace}\left\{\mathbf{Q}^{-1}(\alpha, \beta) \mathbf{H}_{\mathbf{a}}^{T} \mathbf{H}_{\mathbf{a}}\right\},
\end{aligned}
$$

where $\mathbf{Q}(\alpha, \beta)=\alpha(m-1) \mathbf{D}_{\mathbf{a}}^{T} \mathbf{D}_{\mathbf{a}}+\beta(m-1) \mathbf{H}_{\mathbf{a}}^{T} \mathbf{H}_{\mathbf{a}}$ and no a priori information about the parameters is included. Consequently, the regularization parameter is obtained for every iteration as $\lambda(m)=\alpha(m) / \beta(m)$.

Nevertheless, computing the inverse of the matrix $\mathbf{Q}(\alpha, \beta)$ for relative medium sized images turns out a heavy task in terms of computational cost. For that reason, we approximate the second term of (46) considering block circulant matrices also for the aperiodic and truncated models. It means that we can efficiently process the matrix inversion via a 2D FFT, based on the frequency properties of the circulant model. In any case, an iterative method could have been also used to compute $\mathbf{Q}^{-1}(\alpha, \beta)$ without relying on circulant matrices [30].

4.5.2. $\ell_{1}$ Regularizer. In search of another Bayesian fashion solution for $\lambda$, but now applied to the TV regularization problem, we come across the proposed analysis of BioucasDias et al. [17]. By using a Gamma prior for $\lambda$, it leads to

$$
\begin{aligned}
\lambda(m) & =\frac{\rho \sigma^{2}}{\operatorname{TV}\{\widehat{\mathbf{x}}(m)\}+\beta}, \\
\rho & =2(\alpha+\theta \cdot L),
\end{aligned}
$$

where $\operatorname{TV}\{\hat{\mathbf{x}}(m)\}$ was previously defined in (30) and $\alpha$, $\beta$ are the respective shape and scale parameters of the Gamma distribution $p(\lambda / \alpha, \beta) \propto \lambda^{\alpha-1} \exp (-\beta \lambda)$. In any case, these two parameters have not such an influence on the computation of $\lambda$ as $\alpha \ll \theta \cdot L$ and $\beta \ll \operatorname{TV}\{\widehat{\mathbf{x}}(m)\}$. Regarding 


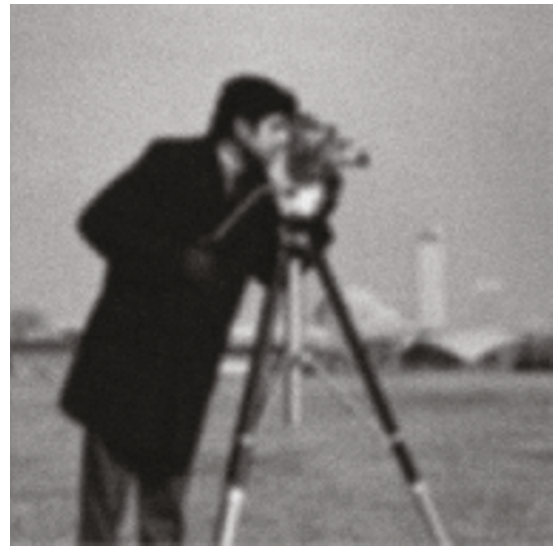

(a)

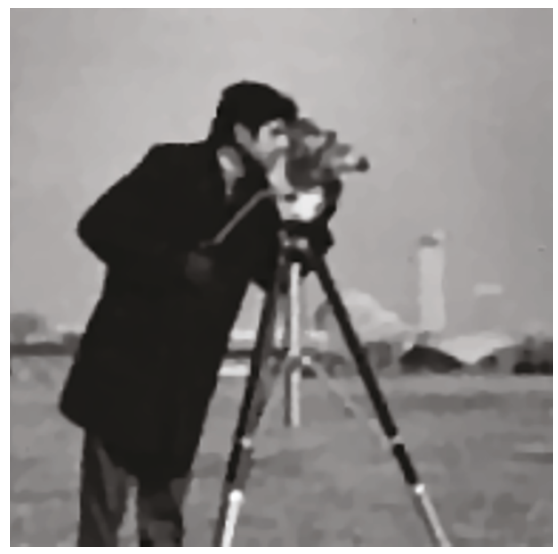

(c)

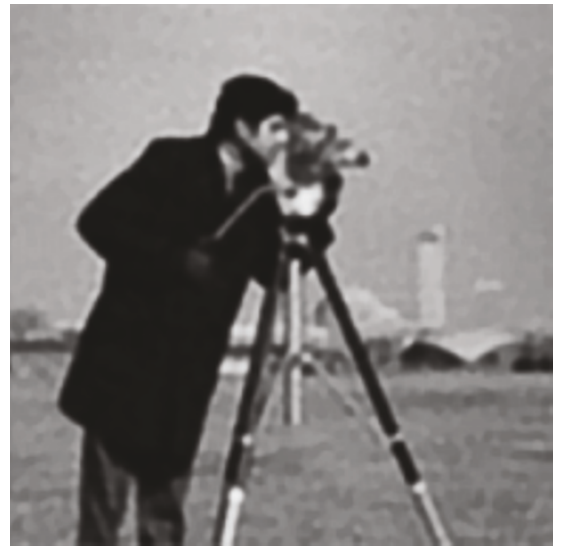

(b)

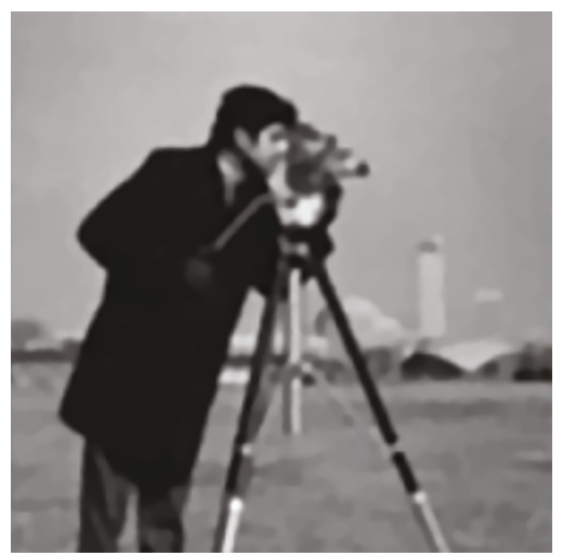

(d)

FIGURE 11: Restoration results from the Cameraman degraded image by Gaussian blur $7 \times 7, \mathrm{BSNR}=20 \mathrm{~dB}$ and CIR model (a). The restored images are shown for (b) $\ell_{1}$-MLP: $\sigma_{e}=15.55$, (c) MM2: $\sigma_{e}=14.73$, and (d) TV1: $\sigma_{e}=15.98$.

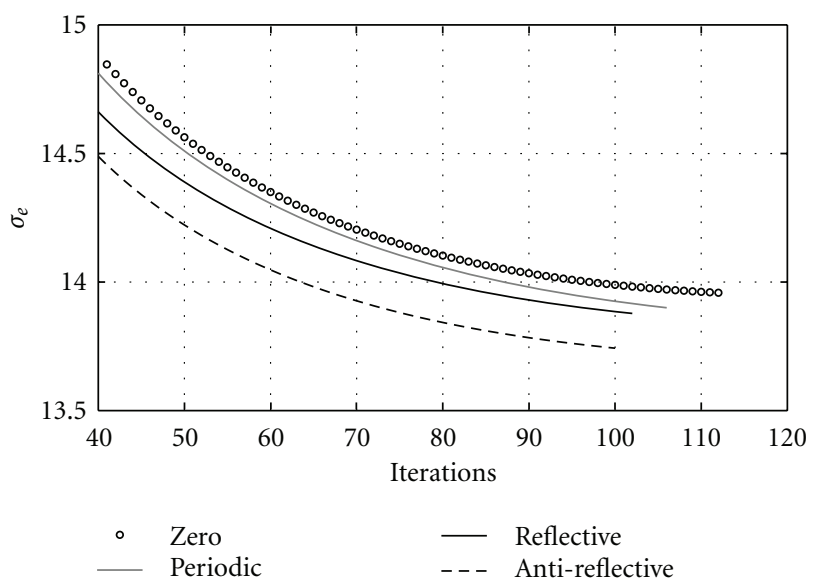

(a)

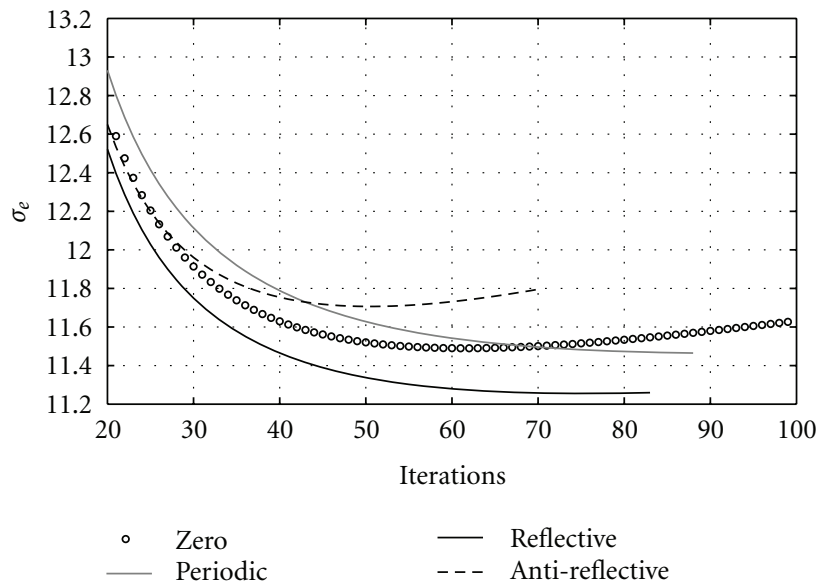

(b)

FIGURE 12: Evolution of the restoration error $\sigma_{e}$ in a $\ell_{2}$-MLP for the boundary conditions: zero, periodic, reflective, and antireflective. Two different plots are shown using a Barbara degraded image by a $7 \times 7$ (a) uniform blur and a (b) diagonal motion blur. 


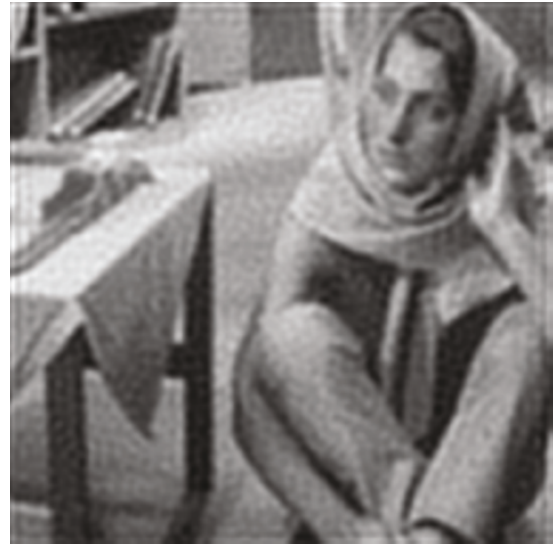

(a)

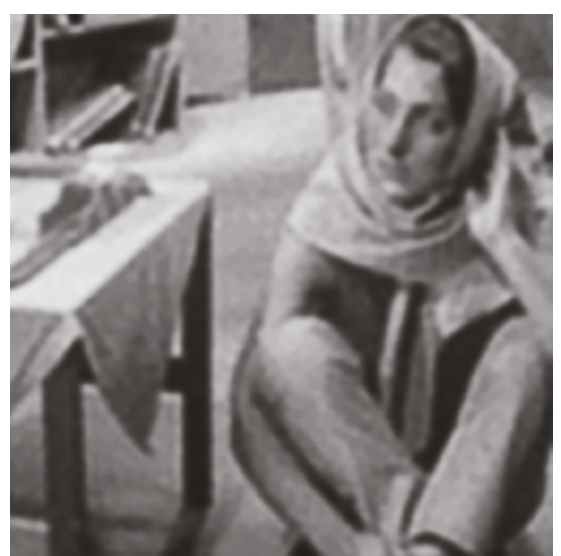

(b)

FIGURE 13: Restoration results from the Barbara degraded image by a Gaussian blur $7 \times 7, \mathrm{BSNR}=20 \mathrm{~dB}$ and zero BC. The restored images are shown for (a) CGLS: $\sigma_{e}=14.06$ and (b) $\ell_{1}$-MLP: $\sigma_{e}=12.56$.

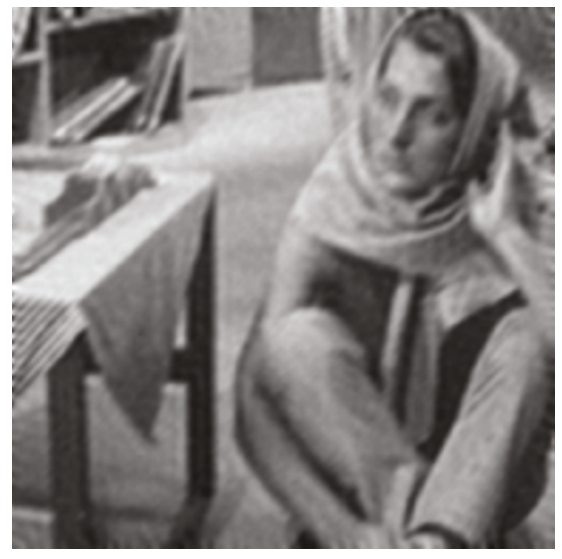

(a)

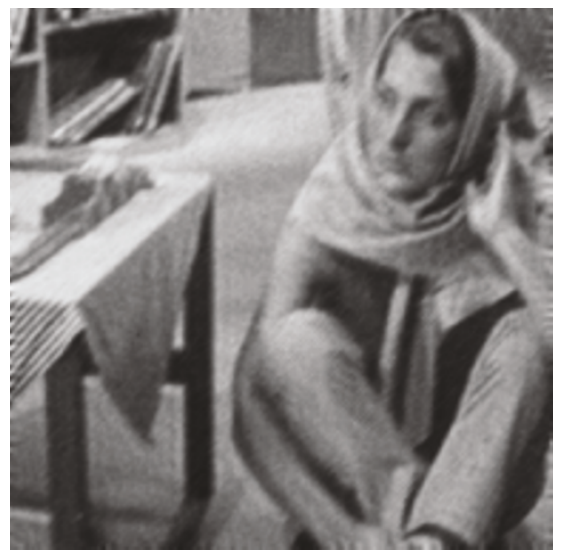

(b)

FIGURE 14: Restoration results from the Barbara degraded image by a diagonal motion blur $7 \times 7, \mathrm{BSNR}=20 \mathrm{~dB}$ and antireflective BC. The restored images are shown for (a) CGLS: $\sigma_{e}=12.29$ and (b) $\ell_{2}$-MLP: $\sigma_{e}=11.80$.

the constant $0<\theta<1$, it is adjusted to get better results of the algorithm and we will provide a heuristic value on a trial and error basis.

\section{Experimental Results}

A number of experiments have been performed with the proposed MLP using several standard images and PSFs, some of which are presented here. The aim is to test the restoration results and the border regeneration properties when considering a real situation of deblurring (truncated model). Moreover, we will evaluate the results when assumed different boundary conditions in the observed image. Let us refer to the truncated, aperiodic and circulant models as TRU, APE and CIR henceforth.

Figure 5 depicts the original Lena image $256 \times 256$ in size of Experiment 1 which is blurred according to those three degradations models. We can observe the zero truncation of borders in TRU, the expansion of the aperiodic convolution of APE and the circular assumption of boundaries in CIR (review Figure 1). We note here the larger size $\widetilde{L}$ of APE and TRU against to the original size $L$ of CIR. Nonetheless, it should be remarked that the TRU image of Figure 5(a) corresponds to the model $\mathbf{y}_{\text {tru }}$ of (6) and the real observed image $\mathbf{y}_{\text {real }}$ is actually the region defined in FOV, that is, $250 \times 250$. Zeros outside the FOV are merely related to the MLP, but not to the real blurred data.

Let us recall that our algorithm is divided into two different implementations depending on the regularization term of $E(m)$, either Tikhonov or TV. Then, we particularize the filter mask $\mathbf{d}$ of the operator $\mathbf{D}$ by means of the Laplace operator with the $\ell_{2}$ regularizer (7)

$$
\frac{1}{6}\left[\begin{array}{ccc}
1 & 4 & 1 \\
4 & -20 & 4 \\
1 & 4 & 1
\end{array}\right],
$$


TABLE 4: Numerical values of $\sigma_{e}$ for $\ell_{2}$ and $\ell_{1}$ regularizers compared with those obtained when $\lambda$ is estimated according to the algorithms of Section 4.5. The results are divided into the degradation model TRU, APE and CIR, as well as the filters $\mathbf{h}_{1}$ and $\mathbf{h}_{2}$.

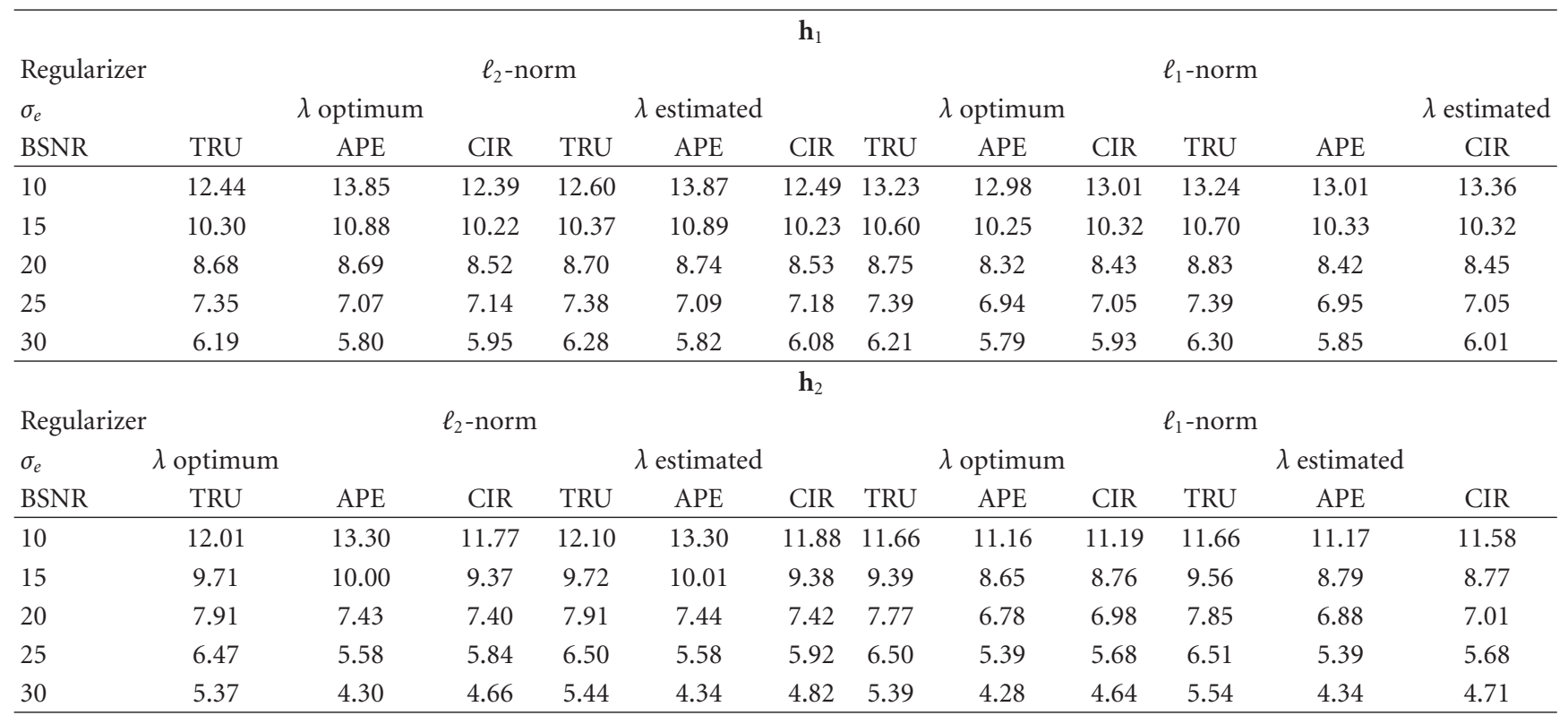

TABle 5: Numerical results obtained from the degraded image of Figure 9(a), when run the set of restoration methods of the Experiment 2 for TRU, APE, and CIR models.

\begin{tabular}{lccc}
\hline$\sigma_{e}$ & TRU & APE & CIR \\
\hline$\ell_{2}$-MLP & 16.08 & 15.76 & 15.85 \\
$\ell_{1}$-MLP & 15.74 & 15.47 & 15.55 \\
MM1 & - & 15.08 & 14.81 \\
MM2 & - & 14.97 & 14.73 \\
TV1 & - & 16.97 & 15.98 \\
TV2 & - & 17.31 & 16.20 \\
SAR & - & 17.13 & 16.30 \\
REG & - & 17.38 & 16.94 \\
WIE & - & 17.60 & 16.72 \\
\hline
\end{tabular}

whereas the Sobel masks [1] are approached to the horizontal $\mathbf{d}^{\xi}$ and vertical $\mathbf{d}^{\mu}$ gradient filters for the $\ell_{1}$ regularizer (8)

$$
\begin{gathered}
\frac{1}{4}\left[\begin{array}{ccc}
-1 & -2 & -1 \\
0 & 0 & 0 \\
1 & 2 & 1
\end{array}\right] \\
\frac{1}{4}\left[\begin{array}{ccc}
-1 & 0 & 1 \\
-2 & 0 & 2 \\
-1 & 0 & 1
\end{array}\right]
\end{gathered}
$$

respectively. Thus, an analogous support $N=[3 \times 3]$ is considered for both regularizations.

As observed in Figure 4, the neural net under analysis consists of two layers $J=2$, where the bias vectors are ignored and the same log-sigmoid function is applied to both layers. Besides, looking for a tradeoff between good quality results and computational complexity, it is assumed that only two neurons take part in the hidden layer, that is, $S^{1}=2$.

In terms of parameters, we previously commented that the learning speed of the net is set to $\eta=1$ or $\eta=2$ if the original image size $L$ is $128 \times 128$ or $256 \times 256$, respectively. On the other hand, the determination of a proper regularization parameter $\lambda$ relies on the Bayesian approaches of Section 4.5. Once computed the MLP, it converges not only to a $\widehat{\mathbf{x}}\left(m_{\text {total }}\right)$, but also to a value of $\lambda\left(m_{\text {total }}\right)$. This last value is fixed for a second round of the neural net so that the restoration performs better results. In any case, we will also try to find out the true optimal value $\lambda$ by sweeping over a wide enough range of possible numbers $\left[\lambda_{\min }, \lambda_{\max }\right]$.

The adjustment of the interconnection weights does not require any network training, so the weigh matrices are initialized to zero along with other preliminary parameters such as $\alpha(0)=0$ or $\beta(0)=0$ related to the $\lambda$ parameter of the $\ell_{2}$ regularization.

In the Algorithm section, we set the stopping criteria as a maximum number of 500 iterations (though never reached) or when the relative difference of the restoration error $E(m)$ falls below a threshold of $10^{-3}$ in a temporal window of 10 iterations.

Different signal-to-noise ratios of the blurred image (BSNR) are used in our experiments defined by

$$
\operatorname{BSNR}=10 \log _{10}\left(\frac{\operatorname{var}\{\mathbf{H x}\}}{\sigma^{2}}\right)
$$

where $\operatorname{var}\{\cdot\}$ calculates the variance of the blurred image without noise over the $\tilde{L}$ support and $\sigma^{2}$ denotes the variance of the Gaussian noise. 
In order to measure the performance of our algorithm, the improvement in signal-to-noise ratio (ISNR) can be adopted [2]

$$
\text { ISNR }=10 \log _{10}\left(\frac{\sum_{q=1}^{L}\left(x_{q}-y_{p}\right)^{2}}{\sum_{q=1}^{L}\left(x_{q}-\hat{x}_{q}\right)^{2}}\right),
$$

where $\mathbf{x}=\left\{x_{q}\right\}_{q=1}^{L}, \hat{\mathbf{x}}=\left\{\hat{\mathbf{x}}_{q}\right\}_{q=1}^{L}, \mathbf{y}=\left\{y_{p}\right\}_{p=1}^{\widetilde{L}}$, and the expression of the numerator contrasts the degraded image and the original image in those homologous pixels within the support $L=\left[L_{1} \times L_{2}\right] \subseteq \widetilde{L}$. However, this expression of ISNR involves the blurred image $\mathbf{y}$, and thus, it is sensitive to the degradation model. For our purposes, we will alternatively use the standard deviation $\sigma_{\mathrm{e}}$ of the error image $\mathbf{e}=\hat{\mathbf{x}}-\mathbf{x}$, such that $\sigma_{e}^{2}$ turns out an approach to the average power of the error.

Our proposed MLP scheme was fully implemented in Matlab, being very well suited as all formulae of this paper have been presented on a matrix basis. The complexity of the net can be analyzed in the two stages which describe the algorithm: forward pass (FP) and backward pass (BP). The computation of the gradient $\boldsymbol{\delta}(m)$ in the output layer makes the BP more time consuming, as shown in (23) and (37). In those equations, the product $\operatorname{trunc}\left\{\mathbf{H}_{\mathbf{a}} \widehat{\mathbf{x}}(m)\right\}$ is the most critical term as it requires numerical computations of $O\left(L^{2}\right)$, although the operator $\operatorname{trunc}\{\cdot\}$ is responsible for discarding (zero-fixing) $2\left(M_{1}-1\right) \times 2\left(M_{2}-1\right)$ operations. However, this high computational cost is significantly reduced for the sparsity of $\mathbf{H}_{\mathbf{a}}$, which obtains a performance only related to the number of nonzero elements. Regarding the FP, the two neurons of the hidden layer lead to faster matrix operations of $O(2 L)$.

In terms of convergence, our MLP is based on the simple steepest descent algorithm as defined in (15). Consequently, the time of convergence is usually slow and controlled by the parameter $\eta$. We are aware that other variations on backpropagation may be applied to our MLP such as the conjugate gradient algorithm, which performs significantly better [31].

Finally, we mention that the experiments were run on a $2.4 \mathrm{GHz}$ Intel Core2Duo with $2 \mathrm{~GB}$ of RAM and we have dedicated a subsection to study the time results in the different stages of the MLP. In any case, future researches may try to improve the complexity of the net.

Experiment 1 . We carry out this experiment by taking the Lena image shown in Figure 5, although down sampling it to become $128 \times 128$ pixels in size for simplifying the computational work. Two blur point spread function $\mathbf{h}$ of size $3 \times 3$

$$
h_{1}(i, j)=\frac{1}{9}\left[\begin{array}{lll}
1 & 1 & 1 \\
1 & 1 & 1 \\
1 & 1 & 1
\end{array}\right], \quad h_{2}(i, j)=\frac{1}{16}\left[\begin{array}{lll}
1 & 2 & 1 \\
2 & 4 & 2 \\
1 & 2 & 1
\end{array}\right],
$$

and a Gaussian noise is added from BSNR $=10 \mathrm{~dB}$ to $\mathrm{BSNR}=30 \mathrm{~dB}$, where the effect of regularization is still noticeable.

Figure 6 depicts the evolution of $\sigma_{e}$ against the BSNR for the filters $\mathbf{h}_{1}$ (a) and $\mathbf{h}_{\mathbf{2}}$ (b), respectively, and using the three degradation models under test (truncated, aperiodic, and circulant). Each figure also contains the results of the $\ell_{2}$ and $\ell_{1}$ regularizations by taking the left and right axes of the ordinates. We can observe how different the regularizations behave on the degradations models although global results favor to the TV regularization for low values of the BSNR.

As demonstrated in [32], the pure aperiodic model achieves better results for high-medium BSNR values when the Tikhonov regularizer is applied. However, it is the circulant model which performs better at low-medium BSNR. These models are fictitious because the borders are not actually present in the observed image. Yet, our proposed neural net is able to adapt to the local nature of the problem and achieve very similar results in the truncated model to those obtained by the two other models. We will observe in next subsection that this adaptation is not well suited by other methods of the literature.

Concerning the $\ell_{1}$ regularizer, the plots indicate that the aperiodic model reaches the minimum restoration error over the whole range of BSNR, but not that far from the circulant degradation. Both models give evidence of the better restoration quality obtained when applying a TV regularizer in the MLP compared to the traditional Tikhonov energy function. It restates the benefits viewed in the literature about considering the TV regularization in a deconvolution problem.

Nevertheless, the $\ell_{1}$ regularizer seems to be more sensitive to the truncation of the image borders, as noted in the deviation of $\sigma_{e}$ from the other models. The improvement of TV over Tikhonov in our MLP is thus more significant for the aperiodic and circulant degradations than a in a real truncated situation.

Numerical results of the same experiment are shown in Table 4 over a finite range of BSNR. Let us recall that the critical regularization parameter $\lambda$ is computed according to (46) and (47) for the $\ell_{2}$ and $\ell_{1}$ regularizations, respectively. Regarding $\ell_{2}$, the trace \{\} operator was computed by using the DFT. In addition, Bioucas-Dias suggests in [17] that a reasonable choice for $\theta$ would be around $2 / 3$ and we set that value in our computation for $\ell_{1}$.

These approaches have yielded very good results in this Experiment when compared to the optimum handtuned $\lambda$ in the Table 4 . However, we are aware that there is room to develop another ways of selecting the regularization parameter, if we aim to extend it for a large range of experiments.

We also note here that the chosen learning speed $\eta=1$ shows lower sensitivity to the restoration error $\sigma_{e}$ than that of the parameter $\lambda$. An example is illustrated in Figure 7 revealing the evolution of $\sigma_{e}$ against both parameters such that $\left(\ell_{2}, \mathbf{h}_{1}, \mathrm{TRU}, 20 \mathrm{~dB}\right)$.

In a short range of $\lambda$ the value of $\sigma_{e}$ changes more rapidly than when going through the learning speed $\eta$. This statement is more obvious when checking the shape of 
TABLE 6: Numerical values of $\sigma_{e}$ obtained for the different boundary conditions including the truncated model in our MLP and the CGLS algorithm. The results are divided into three $7 \times 7$ degradation filters: uniform, Gaussian, and diagonal motion blurs.

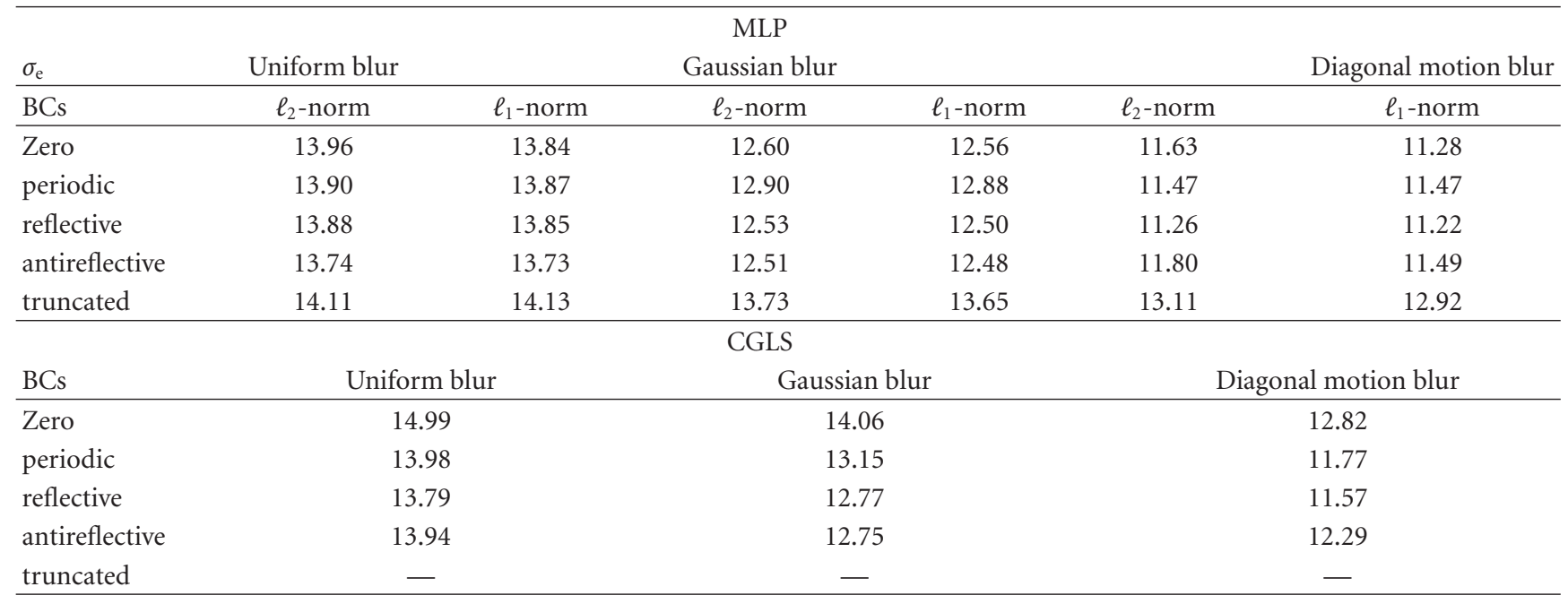

TABLE 7: Time complexity of the MLP for a truncated model using the $256 \times 256$ Barbara image with a $7 \times 7$ Gaussian blur.

\begin{tabular}{lcccc}
\hline Time $(\mathrm{ms})$ & \multicolumn{2}{c}{ Forward } & \multicolumn{2}{c}{ Backward } \\
\hline$m$ & Layer 1 & Layer 2 & Layer 1 & Layer 2 \\
\hline 1 & 2.1 & 3.0 & 8.3 & 523.0 \\
2 & 0.6 & 5.1 & 7.8 & 496.6 \\
3 & 1.2 & 4.5 & 8.0 & 502.2 \\
4 & 1.1 & 4.7 & 8.2 & 500.4 \\
5 & 1.2 & 4.8 & 8.2 & 500.5 \\
& & $\ldots$ & \multicolumn{3}{c}{ Total $=39.2 \mathrm{~s}$} \\
\hline$m_{\text {total }}=76$ & & \multicolumn{3}{c}{}
\end{tabular}

the level curves, being almost parallel straight lines for the variation of $\eta$. We have given more luminosity to the curves as the parameter $\sigma_{e}$ increases.

Finally, we examine how our method performs the regeneration of borders for the real model of (5). Let us run the previous TRU experiment of BSNR $=20 \mathrm{~dB}$ but for the original image size $256 \times 256$, a $7 \times 7$ uniform blur and the optimum $\lambda$ parameter. The observed image $y$ is thus truncated to the region $250 \times 250$, being zeros outside. Figure 8 shows the restored images for $\ell_{2}$ and $\ell_{1}$ regularizations overlaying a white broken line to indicate the regenerated borders. From these images, it can be drawn the ability of our MLP to not only recover the truncated pixels of the original image, but also adapt the center of the image to the optimum solution. We can check that the effect of ringing is negligible and the edges are well preserved in both cases. Though less obvious in this example, the $\ell_{1}$ regularizer leads to visually better results compared to the solution of $\ell_{2}$. It will be manifest in the following experiment.

Experiment 2. To further assess the performance of our proposed image restoration algorithm, we have compared the results of the MLP with other recent work of the literature. The test image is now the $256 \times 256$ sized Cameraman image and the blur is a rotationally symmetric Gaussian lowpass filter of size $7 \times 7$ with standard deviation 2. The BSNR is set again to $20 \mathrm{~dB}$ such that the regularization still plays an important role on the restoration. Regarding the regularization parameter $\lambda$, it is chosen empirically to perform the largest ISNR value in our MLP.

We have evaluated the researches of Bioucas-Dias on the majorization-minimization approach to the TV-based deconvolution. Specifically, we have taken his algorithms presented $[16,17]$, which we will name henceforth as MM1 and MM2, respectively. On the other hand, the hierarchical Bayesian framework utilizing variational distributions of Molina has been also referenced as comparative results. Two versions of the TV algorithm were presented in [18] which will be denoted by TV1 and TV2 in this paper. We have assembled and run the Matlab codes which the authors have gratefully provided us according to the default parameters. In TV1 and TV2, we have particularized them not to have prior information about the hyperparameters, and, therefore, the confidence parameters $\gamma_{\alpha}$ and $\gamma_{\beta}$ have been set to zero. In addition, all the methods have used their respective algorithms to select the parameter $\lambda$.

In order to better appreciate the improvements achieved with respect to the state of art, we have also included traditional algorithms such as the maximum a posteriori analysis of Molina for the simultaneous autoregressive model (SAR) [29], or the Matlab built-in deblurring methods corresponding to the regularized and the Wiener filters, denoted respectively by REG and WIE hereafter.

From the very beginning of this paper, we have commented that most of the work in the literature assumes a circulant model to deal with the restoration problem (that is the case for the previous methods). However, when considering the truncated model as depicted in Figure 9(a), none of those methods works properly and the results are awfully corrupted by the side effect of the zero boundaries. Even if using the real observed image defined in the $250 \times 250$ 
region FOV, the restoration keeps unpleasant artifacts due to the absence of borders assumptions. Look at the ringing lines appeared up and down in the restored image of Figure 10, when using MM1 in the truncated model by cropping the center of the TRU image.

On the contrary, our neural net is very well suited to face the nonlinearity of the zero truncation. It achieves to restore the observed image according to the energy minimization strategy and, furthermore, the lost borders are regenerated. Figures 9(b) and 9(c) illustrate the outcome of the MLP for the $\ell_{2}$ and $\ell_{1}$ regularizations, respectively. Let us point out that the use of the TV regularizer now outperforms clearly the results of the Tikhonov method over the MLP. The edges are noticeably preserved and it besides reduces the artifacts of the noisy observed image. The borders are seamlessly recovered up to the original size $256 \times 256$ and, at the same time, the center of the image is successfully restored and the ringing effect is negligible. The subjective aspect of these results confirms the good performance of our algorithms in a realistic deblurring problem.

In any case, we want to fairly compare the results of those methods for the degradation model which they are actually prepared. So, we run the same experiment but using the circulant model observed in Figure 11(a). Numerical values of the restoration error $\sigma_{e}$ (see Table 5 within CIR column) show the best results obtained by the MM algorithms and followed immediately after by those of our MLP. However, when we have a look to the restored images of Figure 11(c), we notice the overemphasized edges of the MM2 method compared to the more natural aspect of the $\ell_{1}$-MLP. Since we consider a relatively noisy image $\mathrm{BSNR}=20 \mathrm{~dB}$, the other methods tend to oversmooth the results for removing noise artifacts and highlight the edges simultaneously as seen in Figures 11(c) and 11(d).

From these results, we can deduce that our proposed method is also a good reference in the circulant model, obtaining a successful visual aspect with preserve of edges and an acceptable level of denoising (mainly for $\ell_{1}$ regularizer).

Experiment 3. So far, we have focused on the ability of our MLP to reduce the artifacts appeared in a restoration problem with real observed images (5). Now, we aim to check the response of our algorithm when considering the different boundary conditions introduced in Section 2: zero, periodic, reflective and antireflective. It is expected that the ringing effects due to the discontinuities of the boundaries are significantly reduced as done for the truncation of borders in the real model.

To implement every BC, we incorporate the RestoreTools (http://www.mathcs.emory.edu/ nagy/RestoreTools) library into our development patched with the antireflective modification (http://scienze-como.uninsubria.

it/mdonatelli/). RestoreTools facilitates the implementation by providing functions to efficiently implement matrixvector multiplications when assumed every BC. Consequently, we can particularize our algorithm by constructing the matrixes $\mathbf{H}$ and $\mathbf{D}$ adapted to the boundary conditions and removing the operator trunc $\{\cdot\}$ from all the formulae.
The matrixes are all set to square dimensions and then $\widetilde{L}=L$.

In this third experiment, we use the $256 \times 256$ sized Barbara image based on the significant features than can be found at the edge of its viewable region. The $7 \times 7$ uniform and Gaussian blurring operators are retrieved from the previous examples and besides a diagonal motion blur of the same size is considered in our study. Regarding the Gaussian noise, we keep $20 \mathrm{~dB}$ of BSNR in search of a noticeable regularization process using the optimum $\lambda$ parameter.

Let us analyze the impact of each BC on the MLP looking at the evolution of the restoration error $\sigma_{e}$ as the neural net iterates. The plot of $\sigma_{e}$ against iterations is shown in Figure 12 where the uniform blur (a) and the diagonal motion blur (b) are restored by a $\ell_{2}$-MLP. As expected by the literature [7], the reflective and mostly the antireflective boundary conditions also outperforms in our algorithm.

Actually, we aim to compare the ability of our MLP to reduce the ringing artifacts when the same BCs are applied to other methods. So, we take the implementation of the conjugate gradient algorithm with Tikhonov regularization in the RestoreTools library (CGLS). We do not consider any preconditioner in this approach, but we select the optimal regularization parameter. Table 6 outlines the values of $\sigma_{e}$ obtained for our MLP and the CGLS algorithm in every combination $\mathrm{BC}$ and degradation filter. The results of this table reveal the adaptation of the MLP to the local nature of the problem, yielding better results than the CGLS method. Furthermore, our net achieves reasonable restoration errors in a real problem without borders (truncated BC), while the results of CGLS are completely corrupted by the effect of the zero truncation in (5).

If we have a look to the restored images, we confirm in Figure 13 how the MLP makes the ringing effect negligible for a Gaussian blur and zero BC (b); however, the CGLS approach leads to a corrupted image by the discontinuity of the boundary and the noise intensity (a). Regarding the antireflective boundary condition, we find in Figure 14 examples of the restored images for the diagonal motion blur of both methods. We can observe herein the better visual aspect achieved by the MLP (b) adapting the center of the image to the optimum solution and preserving the information of edges.

In conclusion, our MLP is also well suited to deal with the artifacts of a typical restoration problem with BCs, apart from yielding a successful solution when the real truncated model is considered.

5.1. Time Complexity Experiment. To have an idea of the complexity of our net, we have measured the elapsed time in the different stages of the MLP. Specifically, we have used the previous $256 \times 256$ sized Barbara image degraded by a $7 \times 7$ Gaussian blur and $20 \mathrm{~dB}$ of BSNR in a real truncated model (5).

Table 7 shows the results obtained in both layers for the FP and BP. As expected, the backward pass in the output layer is the bottleneck of the MLP. 


\section{Concluding Remarks}

In this paper, we have presented the implementation of a method for image restoration in real conditions, that is to say, an observed image where the borders outside the field of view (FOV) have been truncated. The idea is to apply a deterministic regularization function, particularized to the $\ell_{2}$ and $\ell_{1}$ norms in an iterative minimization of a MLP (Multilayer perceptron) neural net. An inherent backpropagation algorithm has been developed in order to regenerate the lost borders, while adapting the center of the image to the optimum linear solution (the ringing artifact thus being negligible).

The proposed restoration scheme has been validated by means of several tests. As a result, we can conclude the ability of our neural net to deal with the nonlinearity of border truncation, in contrast to other state-of-art methods which are mostly based on the assumption of circular convolution. The total variation (TV) regularizer achieves visually better results, preserving the edges and reducing the artifacts of the noisy observed image. Apart from the aforementioned regeneration of borders, our algorithm has also achieved good performance when compared with other recent researches using boundary conditions.

\section{Appendix}

\section{Derivation of $(22)$}

By replacing the definition of $E(m)$ from (17), it is immediately obtained that

$$
\begin{aligned}
\frac{\partial E(m)}{\partial \widehat{\mathbf{x}}(m)} & =\frac{1}{2} \frac{\partial\|\mathbf{e}(m)\|_{2}^{2}}{\partial \widehat{\mathbf{x}}(m)}+\frac{1}{2} \lambda \frac{\partial\|\mathbf{r}(m)\|_{2}^{2}}{\partial \widehat{\mathbf{x}}(m)} \\
& =\frac{1}{2} \frac{\partial \mathbf{e}(m)}{\partial \widehat{\mathbf{x}}(m)} \frac{\partial\|\mathbf{e}(m)\|_{2}^{2}}{\partial \mathbf{e}(m)}+\frac{1}{2} \lambda \frac{\partial \mathbf{r}(m)}{\partial \widehat{\mathbf{x}}(m)} \frac{\partial\|\mathbf{r}(m)\|_{2}^{2}}{\partial \mathbf{r}(m)} .
\end{aligned}
$$

As the $\ell_{2}$ norm is defined by $\|\mathbf{z}\|_{2}^{2}=\mathbf{z}^{T} \mathbf{z}$ leading to $\partial\|\mathbf{z}\|_{2}^{2} / \partial \mathbf{z}=2 \mathbf{z}$, then

$$
\frac{\partial E(m)}{\partial \widehat{\mathbf{x}}(m)}=\frac{\partial \mathbf{e}(m)}{\partial \widehat{\mathbf{x}}(m)} \mathbf{e}(m)+\lambda \frac{\partial \mathbf{r}(m)}{\partial \widehat{\mathbf{x}}(m)} \mathbf{r}(m) .
$$

Both vectors $\mathbf{e}(m)$ and $\mathbf{r}(m)$ of (16) and can be respectively, substituted into (A.2) as follows:

$$
\begin{aligned}
\frac{\partial E(m)}{\partial \widehat{\mathbf{x}}(m)}= & \frac{\partial\left(-\operatorname{trunc}\left\{\mathbf{H}_{\mathbf{a}} \widehat{\mathbf{x}}(m)\right\}\right)}{\partial \widehat{\mathbf{x}}(m)} \mathbf{e}(m) \\
& +\lambda \frac{\partial\left(\operatorname{trunc}\left\{\mathbf{D}_{\mathbf{a}} \widehat{\mathbf{x}}(m)\right\}\right)}{\partial \widehat{\mathbf{x}}(m)} \mathbf{r}(m),
\end{aligned}
$$

where the degraded image $\mathbf{y}$ is removed as no explicit dependency with $\widehat{\mathbf{x}}(m)$ is considered. Regarding the operator $\operatorname{trunc}\{\cdot\}$, it is already included within the multiplicative terms $\mathbf{e}(m)$ and $\mathbf{r}(m)$; therefore, those elements in the differentiation terms where the operator must work (zerofixing), it turns out that $\mathbf{e}(m)$ and $\mathbf{r}(m)$ are zeros as well. So, we can directly remove the operator $\operatorname{trunc}\{\cdot\}$ in (A.3) with no further consequences

$$
\frac{\partial E(m)}{\partial \widehat{\mathbf{x}}(m)}=\frac{\partial\left(-\mathbf{H}_{\mathbf{a}} \widehat{\mathbf{x}}(m)\right)}{\partial \widehat{\mathbf{x}}(m)} \mathbf{e}(m)+\frac{\partial\left(\mathbf{D}_{\mathbf{a}} \widehat{\mathbf{x}}(m)\right)}{\partial \widehat{\mathbf{x}}(m)} \mathbf{r}(m) .
$$

We can finally obtain by matrix differentiation that

$$
\frac{\partial E(m)}{\partial \hat{\mathbf{x}}(m)}=-\mathbf{H}_{\mathbf{a}}^{T} \mathbf{e}(m)+\mathbf{D}_{\mathbf{a}}^{T} \mathbf{r}(m) .
$$

\section{Acknowledgments}

The authors would like to thank Dr. J. Bioucas-Dias in the Deparment of Electrical and Computer Engineering, Technical University of Lisbon, Portugal and Dr. R. Molina in the Department of Computer Science and Artificial Intelligence, University of Granada, Spain, for their fruitful discussions in the course of this work and for providing their respective Matlab codes of the restoration methods in Experiment 2.

\section{References}

[1] R. C. González and R. E. Woods, Digital Image Processing, Prentice Hall, Upper Saddle River, NJ, USA, 3rd edition, 2008.

[2] M. R. Banham and A. K. Katsaggelos, "Digital image restoration," IEEE Signal Processing Magazine, vol. 14, no. 2, pp. 2441, 1997.

[3] A. C. Bovik, Handbook of Image \& Video Processing, Elsevier, Amsterdam, The Netherlands, 2nd edition, 2005.

[4] T. F. Chan and J. Shen, "Image processing and analysis variational, PDE, wavelet and stochastic methods," in Frontiers in Applied Mathematics, SIAM, Philadelphia, Pa, USA, 2005.

[5] J. W. Woods, J. Biemond, and A. M. Tekalp, "Boundary value problem in image restoration," in Proceedings of the IEEE International Conference on Acoustics, Speech, and Signal Processing (ICASSP '85), vol. 10, pp. 692-695, 1985.

[6] D. Calvetti and E. Somersalo, "Statistical elimination of boundary artefacts in image deblurring," Inverse Problems, vol. 21, no. 5, pp. 1697-1714, 2005.

[7] M. Donatelli, C. Estatico, A. Martinelli, and S. SerraCapizzano, "Improved image deblurring with anti-reflective boundary conditions and re-blurring," Inverse Problems, vol. 22, no. 6, article no. 008, pp. 2035-2053, 2006.

[8] R. Liu and J. Jia, "Reducing boundary artifacts in image deconvolution," in Proceedings of the IEEE International Conference on Image Processing (ICIP '08), pp. 505-508, October 2008.

[9] J. K. Paik and A. K. Katsaggelos, "Image restoration using a modified Hopfield network," IEEE Transactions of Image Processing, vol. 1, no. 1, pp. 49-63, 1992.

[10] Y. Sun, "Hopfield neural network based algorithms for image restoration and reconstruction-part II: performance analysis," IEEE Transactions on Signal Processing, vol. 48, no. 7, pp. 2119-2131, 2000.

[11] Y. B. Han and L. N. Wu, "Image restoration using a modified hopfield neural network of continuous state change," Signal Processing, vol. 12, no. 3, pp. 431-435, 2004.

[12] S. W. Perry and L. Guan, "Weight assignment for adaptive image restoration by neural networks," IEEE Transactions on Neural Networks, vol. 11, no. 1, pp. 156-170, 2000.

[13] H.-S. Wong and L. Guan, "A neural learning approach for adaptive image restoration using a fuzzy model-based network 
architecture," IEEE Transactions on Neural Networks, vol. 12, no. 3, pp. 516-531, 2001.

[14] H. Zhang, Y. Wu, and Q. Peng, "Image restoration using hopfield neural network based on Total Variational model," in Proceedings of the Second International Symposium on Neural Networks (ISNN '05), vol. 3497 of Lecture Notes in Computer Science, pp. 735-740, June 2005.

[15] Y.-D. Wu, Y. Sun, H.-Y. Zhang, and S.-X. Sun, "Variational PDE based image restoration using neural network," IET Image Processing, vol. 1, no. 1, pp. 85-93, 2007.

[16] J. M. Bioucas-Dias, M. A. T. Figueiredo, and J. P. Oliveira, "Total variation-based image deconvolution: a majorizationminimization approach," in Proceedings of the IEEE International Conference on Acoustics, Speech and Signal Processing (ICASSP '06), pp. II861-II864, May 2006.

[17] J. P. Oliveira, J. M. Bioucas-Dias, and M. A. T. Figueiredo, "Adaptive total variation image deblurring: a majorizationminimization approach," Signal Processing, vol. 89, no. 9, pp. 2479-2493, 2009.

[18] R. Molina, J. Mateos, and A. K. Katsaggelos, "Blind deconvolution using a variational approach to parameter, image, and blur estimation," IEEE Transactions on Image Processing, vol. 15, no. 12, pp. 3715-3727, 2006.

[19] E. Bermiés, G. Cisneros, and M. Capella, "Truncated edges estimation using MLP neural nets applied to regularized image restoration," in Proceedings of the International Conference on Image Processing (ICIP '02), pp. 341-344, September 2002.

[20] G. Peyré, S. Bougleux, and L. Cohen, "Non-local regularization of inverse problems," in Proceedings of the 10th European Conference on Computer Vision (ECCV '08), vol. 5304 of Lecture Notes in Computer Science, pp. 57-68, 2008.

[21] M. Mignotte, "A non-local regularization strategy for image deconvolution," Pattern Recognition Letters, vol. 29, no. 16, pp. 2206-2212, 2008

[22] M. A. Santiago, G. Cisneros, and E. Bernués, "Iterative desensitisation of image restoration filters under wrong PSF and noise estimates," Eurasip Journal on Advances in Signal Processing, vol. 2007, Article ID 72658, 18 pages, 2007.

[23] M. Bertero and P. Boccacci, Introduction to Inverse Problems in Imaging, Institute of Physics Publishing, 1998.

[24] M. K. Ng, R. H. Chan, and W.-C. Tang, "Fast algorithm for deblurring models with Neumann boundary conditions," SIAM Journal of Scientific Computing, vol. 21, no. 3, pp. 851866, 1999.

[25] L. I. Rudin, S. Osher, and E. Fatemi, "Nonlinear total variation based noise removal algorithms," Physica D, vol. 60, no. 1-4, pp. 259-268, 1992.

[26] K. Brandt and M. Sysking, "The Matrix Cookbook," 2008, http://matrixcookbook.com.

[27] C. A. Felippa, "Introduction to finite element methods," 2009, http://www.colorado.edu/engineering/cas/courses.d/IFEM.d/.

[28] C. R. Vogel, "Computational methods for inverse problems," in Frontiers in Applied Mathematics, SIAM, 2002.

[29] R. Molina, A. K. Katsaggelos, and J. Mateos, "Bayesian and regularization methods for hyperparameter estimation in image restoration," IEEE Transactions on Image Processing, vol. 8, no. 2, pp. 231-246, 1999.

[30] I. T. Dimov, T. T. Dimov, and T. V. Gurov, "A new iterative Monte Carlo approach for inverse matrix problem," Journal of Computational and Applied Mathematics, vol. 92, no. 1, pp. 15-35, 1998.

[31] M. T. Hagan, H. B. Demuth, and M. H. Beale, Neural Network Design, PWS Publishing, Boston, Mass, USA, 1996.
[32] Z. Mou-yan and R. Unbehauen, "Circulant and aperiodic models of deconvolution: a comparison," Signal Processing, vol. 64, no. 2, pp. 185-192, 1998. 